\title{
FOLDING PROCEDURE FOR NEWTON-OKOUNKOV POLYTOPES OF SCHUBERT VARIETIES
}

\author{
NAOKI FUJITA
}

\begin{abstract}
The theory of Newton-Okounkov polytopes is a generalization of that of Newton polytopes for toric varieties, and it gives a systematic method of constructing toric degenerations of a projective variety. In the case of Schubert varieties, their Newton-Okounkov polytopes are deeply connected with representation theory. Indeed, Littelmann's string polytopes and Nakashima-Zelevinsky's polyhedral realizations are obtained as Newton-Okounkov polytopes of Schubert varieties. In this paper, we apply the folding procedure to a Newton-Okounkov polytope of a Schubert variety, which relates NewtonOkounkov polytopes of Schubert varieties of different types. As an application of this result, we obtain a new interpretation of Kashiwara's similarity of crystal bases.
\end{abstract}

\section{Contents}

1. Introduction

2. Littelmann's string polytopes and Nakashima-Zelevinsky's polyhedral realizations

3. Perfect bases and Newton-Okounkov polytopes

4. Orbit Lie algebras

5. Fixed point Lie subalgebras

6. Relation with similarity of crystal bases 19

Appendix A. Case of affine Lie algebras $\quad 21$

References

\section{InTRODUCTION}

This paper is devoted to the study of the folding procedure for a Newton-Okounkov polytope of a Schubert variety. The theory of Newton-Okounkov polytopes was introduced by Okounkov [37, 38], and afterward developed independently by Kaveh-Khovanskii [22] and by Lazarsfeld-Mustata [26]. It is a generalization of the theory of Newton polytopes for toric varieties to arbitrary projective varieties, and it gives a systematic method of constructing toric degenerations by [1, Theorem 1] (see also [12]). In the case of Schubert varieties, their Newton-Okounkov polytopes include some representation-theoretic polytopes such as Littelmann's string polytopes [21], Nakashima-Zelevinsky's polyhedral realizations [9], and Feigin-Fourier-Littelmann-Vinberg's polytopes [5, 24]; in addition, Lusztig's parametrization of the canonical basis also appears in the theory of Newton-Okounkov polytopes (see [4]). In this paper, we study Littelmann's string polytopes and Nakashima-Zelevinsky's polyhedral realizations, and obtain relations among these polytopes for Schubert varieties of different types.

To be more precise, let $\mathfrak{g}$ be a simply-laced simple Lie algebra, $\mathfrak{t} \subset \mathfrak{g}$ a Cartan subalgebra, $P_{+} \subset \mathfrak{t}^{*}$ the set of dominant weights for $\mathfrak{g}$, and $\omega: I \rightarrow I$ a Dynkin diagram automorphism, where $I$ is an index set for the vertices of the Dynkin diagram. In this paper, for technical reasons, we always assume that any two vertices of the Dynkin diagram in the same $\omega$-orbit are not joined. Such an $\omega$ induces a Lie algebra automorphism $\omega: \mathfrak{g} \stackrel{\sim}{\rightarrow} \mathfrak{g}$, which preserves the Cartan subalgebra $\mathfrak{t}$. We know that the fixed point Lie subalgebra $\mathfrak{g}^{\omega}:=\{x \in \mathfrak{g} \mid \omega(x)=x\}$ is also a simple Lie algebra. Fix a complete set $\breve{I}$ of representatives for the $\omega$-orbits in $I$; the set $\breve{I}$ is identified with an index set for the vertices of the Dynkin diagram of $\mathfrak{g}^{\omega}$. Then, there exists a natural injective group homomorphism $\Theta: \breve{W} \hookrightarrow W$ from the Weyl group of $\mathfrak{g}^{\omega}$ to that of $\mathfrak{g}$. If $\mathbf{i}=\left(i_{1}, \ldots, i_{r}\right) \in \breve{I}^{r}$ is a reduced word for $w \in \breve{W}$, then

$$
\Theta(\mathbf{i}):=\left(i_{1,1}, \ldots, i_{1, m_{i_{1}}}, \ldots, i_{r, 1}, \ldots, i_{r, m_{i_{r}}}\right) \in I^{m_{i_{1}}+\cdots+m_{i_{r}}}
$$

Date: November 5, 2018.

2010 Mathematics Subject Classification. Primary 17B37; Secondary 05E10, 14M15, 14 M25.

Key words and phrases. Newton-Okounkov body, Schubert variety, Crystal basis, Orbit Lie algebra, Fixed point Lie subalgebra.

The work was partially supported by Grant-in-Aid for JSPS Fellows (No. 16J00420). 
is a reduced word for $\Theta(w)$, where we set $m_{i}:=\min \left\{k \in \mathbb{Z}_{>0} \mid \omega^{k}(i)=i\right\}$ for $i \in \breve{I}$ and $i_{k, l}:=\omega^{l-1}\left(i_{k}\right)$ for $1 \leq k \leq r, 1 \leq l \leq m_{i_{k}}$. Let $\omega^{*}: \mathfrak{t}^{*} \stackrel{\sim}{\rightarrow} \mathfrak{t}^{*}$ be the dual of the $\mathbb{C}$-linear automorphism $\omega: \mathfrak{t} \stackrel{\sim}{\rightarrow} \mathfrak{t}$, and set $\left(\mathfrak{t}^{*}\right)^{0}:=\left\{\lambda \in \mathfrak{t}^{*} \mid \omega^{*}(\lambda)=\lambda\right\}$. Note that an element $\lambda \in P_{+} \cap\left(\mathfrak{t}^{*}\right)^{0}$ naturally induces a weight $\hat{\lambda}$ for $\mathfrak{g}^{\omega}$. Now, for $w \in \breve{W}$ and $\lambda \in P_{+} \cap\left(\mathfrak{t}^{*}\right)^{0}$, let $X(w)$ (resp., $X(\Theta(w))$ ) be the corresponding Schubert variety, and $\mathcal{L}_{\hat{\lambda}}$ (resp., $\mathcal{L}_{\lambda}$ ) the corresponding line bundle on $X(w)$ (resp., $X(\Theta(w))$ ). Also, let $\Delta_{\mathbf{i}}^{(\hat{\lambda}, w)}, \Delta_{\Theta(\mathbf{i})}^{(\lambda, \Theta(w))}$ (resp., $\widetilde{\Delta}_{\mathbf{i}}^{(\hat{\lambda}, w)}, \widetilde{\Delta}_{\Theta(\mathbf{i})}^{(\lambda, \Theta)}$ ) denote Littelmann's string polytopes (resp., NakashimaZelevinsky's polyhedral realizations) corresponding to $w \in \breve{W}$ and $\lambda \in P_{+} \cap\left(\mathfrak{t}^{*}\right)^{0}$; see Definition 2.8 for the definitions. Kaveh [21] (resp., the author and Naito [9]) proved that

$$
\begin{aligned}
\Delta_{\mathbf{i}}^{(\hat{\lambda}, w)} & =-\Delta\left(X(w), \mathcal{L}_{\hat{\lambda}}, v_{\mathbf{i}}, \tau_{\hat{\lambda}}\right), \Delta_{\Theta(\mathbf{i})}^{(\lambda, \Theta(w))}=-\Delta\left(X(\Theta(w)), \mathcal{L}_{\lambda}, v_{\Theta(\mathbf{i})}, \tau_{\lambda}\right) \\
\left(\text { resp. }, \widetilde{\Delta}_{\mathbf{i}}^{(\hat{\lambda}, w)}\right. & \left.=-\Delta\left(X(w), \mathcal{L}_{\hat{\lambda}}, \tilde{v}_{\mathbf{i}}, \tau_{\hat{\lambda}}\right), \widetilde{\Delta}_{\Theta(\mathbf{i})}^{(\lambda, \Theta(w))}=-\Delta\left(X(\Theta(w)), \mathcal{L}_{\lambda}, \tilde{v}_{\Theta(\mathbf{i})}, \tau_{\lambda}\right)\right)
\end{aligned}
$$

for specific valuations $v_{\mathbf{i}}, v_{\Theta(\mathbf{i})}\left(\right.$ resp., $\left.\tilde{v}_{\mathbf{i}}, \tilde{v}_{\Theta(\mathbf{i})}\right)$ and specific sections $\tau_{\hat{\lambda}}, \tau_{\lambda}$, where the sets on the righthand side of these equations denote the corresponding Newton-Okounkov polytopes (see Definitions 3.9 and 3.11 for the definitions). The following is the main result of this paper.

Theorem. Define an $\mathbb{R}$-linear surjective map $\Omega_{\mathbf{i}}=\Omega_{\mathbf{i}}^{(\omega)}: \mathbb{R}^{m_{i_{1}}+\cdots+m_{i_{r}}} \rightarrow \mathbb{R}^{r}$ by

$$
\Omega_{\mathbf{i}}\left(a_{1,1}, \ldots, a_{1, m_{i_{1}}}, \ldots, a_{r, 1}, \ldots, a_{r, m_{i_{r}}}\right):=\left(a_{1,1}+\cdots+a_{1, m_{i_{1}}}, \ldots, a_{r, 1}+\cdots+a_{r, m_{i_{r}}}\right) .
$$

Then the following equalities hold:

$$
\begin{aligned}
& \Omega_{\mathbf{i}}\left(\Delta\left(X(\Theta(w)), \mathcal{L}_{\lambda}, v_{\Theta(\mathbf{i})}, \tau_{\lambda}\right)\right)=\Delta\left(X(w), \mathcal{L}_{\hat{\lambda}}, v_{\mathbf{i}}, \tau_{\hat{\lambda}}\right), \text { and } \\
& \Omega_{\mathbf{i}}\left(\Delta\left(X(\Theta(w)), \mathcal{L}_{\lambda}, \tilde{v}_{\Theta(\mathbf{i})}, \tau_{\lambda}\right)\right)=\Delta\left(X(w), \mathcal{L}_{\hat{\lambda}}, \tilde{v}_{\mathbf{i}}, \tau_{\hat{\lambda}}\right) .
\end{aligned}
$$

In our proof of the theorem above, we use another simply-laced simple Lie algebra $\mathfrak{g}^{\prime}$ having a Dynkin diagram automorphism $\omega^{\prime}: I^{\prime} \rightarrow I^{\prime}$ satisfying the following conditions:

$(\mathrm{C})_{1}$ the fixed point Lie subalgebra $\left(\mathfrak{g}^{\prime}\right)^{\omega^{\prime}}$ is isomorphic to the orbit Lie algebra $\breve{\mathfrak{g}}$ associated to $\omega$; this condition implies that the index set $\breve{I}$ for $\breve{\mathfrak{g}}$ is identified with an index set $\breve{I}^{\prime}\left(=\left(\breve{I^{\prime}}\right)\right)$ for $\left(\mathfrak{g}^{\prime}\right)^{\omega^{\prime}}$

$(\mathrm{C})_{2}$ if we set $m_{i}^{\prime}:=\min \left\{k \in \mathbb{Z}_{>0} \mid\left(\omega^{\prime}\right)^{k}(i)=i\right\}, i \in \breve{I}^{\prime}$, then the product $L:=m_{i} \cdot m_{i}^{\prime}$ is independent of the choice of $i \in \breve{I} \simeq \breve{I}^{\prime}$.

Let $\mathbf{i}=\left(i_{1}, \ldots, i_{r}\right) \in \breve{I}^{r} \simeq\left(\breve{I}^{\prime}\right)^{r}$ be a reduced word. It is known that $P_{+} \cap\left(\mathfrak{t}^{*}\right)^{0}$ is identified with the set of dominant weights for the orbit Lie algebra $\breve{\mathfrak{g}}$ associated to $\omega$; let $\breve{\lambda}$ denote the dominant weight for $\breve{\mathfrak{g}}$ corresponding to $\lambda \in P_{+} \cap\left(\mathfrak{t}^{*}\right)^{0}$. Now we define an $\mathbb{R}$-linear injective map $\Upsilon_{\mathbf{i}}=\Upsilon_{\mathbf{i}}^{(\omega)}: \mathbb{R}^{r} \hookrightarrow \mathbb{R}^{m_{i_{1}}+\cdots+m_{i_{r}}}$ by

$$
\Upsilon_{\mathbf{i}}\left(a_{1}, \ldots, a_{r}\right):=(\underbrace{a_{1}, \ldots, a_{1}}_{m_{i_{1}}}, \ldots, \underbrace{a_{r}, \ldots, a_{r}}_{m_{i_{r}}}) .
$$

By using the theory of crystal bases, we see that Littelmann's string polytope (resp., NakashimaZelevinsky's polyhedral realization) for $\breve{\mathfrak{g}}$ with respect to $\breve{\lambda}$ and $\mathbf{i}$ is identified with a slice of $\Delta_{\Theta(\mathbf{i})}^{(\lambda, \Theta)}$ (resp., $\widetilde{\Delta}_{\Theta(\mathbf{i})}^{(\lambda, \Theta(w))}$ ) through $\Upsilon_{\mathbf{i}}$ (see Corollary 4.10 for more details). Hence we obtain the following diagram:

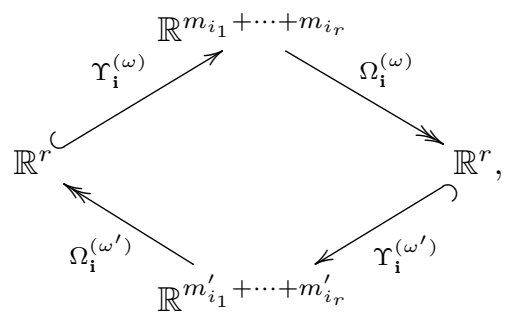

in which the composite maps $\Omega_{\mathbf{i}}^{(\omega)} \circ \Upsilon_{\mathbf{i}}^{(\omega)} \circ \Omega_{\mathbf{i}}^{\left(\omega^{\prime}\right)} \circ \Upsilon_{\mathbf{i}}^{\left(\omega^{\prime}\right)}$ and $\Omega_{\mathbf{i}}^{\left(\omega^{\prime}\right)} \circ \Upsilon_{\mathbf{i}}^{\left(\omega^{\prime}\right)} \circ \Omega_{\mathbf{i}}^{(\omega)} \circ \Upsilon_{\mathbf{i}}^{(\omega)}$ are both identical to $L \cdot \mathrm{id}_{\mathbb{R}^{r}}$, where $L$ is the positive integer in $(\mathrm{C})_{2}$. This diagram plays an important role in our proof of the Theorem above. If $\mathfrak{g}$ is of type $A_{2 n-1}$ and $\omega$ is its Dynkin diagram automorphism of order two, then $\mathfrak{g}^{\omega}$ is of type $C_{n}$ and $\left(\mathfrak{g}^{\prime}, \omega^{\prime}\right)$ is given uniquely by the pair of the simple Lie algebra of type $D_{n+1}$ and its Dynkin diagram automorphism of order two; the fixed point Lie subalgebra $\left(\mathfrak{g}^{\prime}\right)^{\omega^{\prime}}$ is of type $B_{n}$. Thus the diagram above relates Newton-Okounkov polytopes of Schubert varieties of types $A, B, C$, and $D$. A remarkable fact is that the composite map $\Omega_{\mathbf{i}} \circ \Upsilon_{\mathbf{i}}$ is identical to the map coming from a similarity of 
crystal bases. This gives a new interpretation of the similarity of crystal bases in terms of the folding procedure.

This paper is organized as follows. In Section 2, we recall some basic facts about Littelmann's string polytopes and Nakashima-Zelevinsky's polyhedral realizations. In Section 3, we review main results of [9] and [21]. Section 4 is devoted to the study of the folding procedure for crystal bases. In Section 5 , we prove the Theorem above. In Section 6, we study the relation with a similarity of crystal bases. Finally, we mention that our arguments in this paper are naturally extended to symmetrizable KacMoody algebras; in Appendix A, we give the list of nontrivial pairs of automorphisms of simply-laced affine Dynkin diagrams satisfying conditions $(\mathrm{C})_{1}$ and $(\mathrm{C})_{2}$ above.

Acknowledgements. The author is greatly indebted to his supervisor Satoshi Naito for fruitful discussions and numerous helpful suggestions. The author would also like to thank Hironori Oya for suggesting the relation with a similarity of crystal bases.

\section{LitTELMANN'S STRING POLYTOPES AND NAKASHIMA-ZELEVINSKY'S POLYHEDRAL REALIZATIONS}

In this section, we consider Littelmann's string polytopes and Nakashima-Zelevinsky's polyhedral realizations, which are the main objects of our study. We first recall some basic facts about crystal bases, following $[16,17,18,19]$. Let $G$ be a connected, simply-connected simple algebraic group over $\mathbb{C}, \mathfrak{g}$ its Lie algebra, $W$ the Weyl group, $T \subset G$ a maximal torus, and $I$ an index set for the vertices of the Dynkin diagram of $\mathfrak{g}$. Let $\mathfrak{t} \subset \mathfrak{g}$ denote the Lie algebra of $T, \mathfrak{t}^{*}:=\operatorname{Hom}_{\mathbb{C}}(\mathfrak{t}, \mathbb{C})$ the dual space of $\mathfrak{t}$, and $\langle\cdot, \cdot\rangle: \mathfrak{t}^{*} \times \mathfrak{t} \rightarrow \mathbb{C}$ the canonical pairing. Denote by $P \subset \mathfrak{t}^{*}$ the weight lattice for $\mathfrak{g}$, by $P_{+} \subset P$ the set of dominant integral weights, by $\left\{\alpha_{i} \mid i \in I\right\} \subset \mathfrak{t}^{*}$ the set of simple roots, and by $\left\{h_{i} \mid i \in I\right\} \subset \mathfrak{t}$ the set of simple coroots. For an indeterminate $q$, we define $q_{i} \in \mathbb{Q}(q), i \in I$, by:

$$
q_{i}= \begin{cases}q^{3} & \text { if } \mathfrak{g} \text { is of type } G_{2} \text { and } \alpha_{i} \text { is a long root, } \\ q^{2} & \text { if } \mathfrak{g} \text { is of type } B_{n}, C_{n}, n \geq 2, \text { or } F_{4}, \text { and } \alpha_{i} \text { is a long root } \\ q & \text { otherwise. }\end{cases}
$$

Let $U_{q}(\mathfrak{g})$ be the quantized enveloping algebra of $\mathfrak{g}$ over $\mathbb{Q}(q)$ with generators $\left\{e_{i}, f_{i}, t_{i}, t_{i}^{-1} \mid i \in I\right\}$, and $U_{q}\left(\mathfrak{u}^{-}\right)$the $\mathbb{Q}(q)$-subalgebra of $U_{q}(\mathfrak{g})$ generated by $\left\{f_{i} \mid i \in I\right\}$. Denote by $\mathcal{B}(\infty)$ the crystal basis of $U_{q}\left(\mathfrak{u}^{-}\right)$with $b_{\infty} \in \mathcal{B}(\infty)$ the element corresponding to $1 \in U_{q}\left(\mathfrak{u}^{-}\right)$, and by $\tilde{e}_{i}, \tilde{f}_{i}: \mathcal{B}(\infty) \cup\{0\} \rightarrow$ $\mathcal{B}(\infty) \cup\{0\}$ for $i \in I$ the Kashiwara operators.

Definition 2.1. Define a $\mathbb{Q}(q)$-algebra anti-involution $*$ on $U_{q}(\mathfrak{g})$ by:

$$
e_{i}^{*}=e_{i}, f_{i}^{*}=f_{i}, t_{i}^{*}=t_{i}^{-1}
$$

for $i \in I$; we see by [19, Theorem 2.1.1] that this induces an involution $*: \mathcal{B}(\infty) \rightarrow \mathcal{B}(\infty)$, called Kashiwara's involution.

For $\lambda \in P_{+}$, denote by $V_{q}(\lambda)$ the irreducible highest weight $U_{q}(\mathfrak{g})$-module with highest weight $\lambda$ over $\mathbb{Q}(q)$, and by $v_{q, \lambda} \in V_{q}(\lambda)$ the highest weight vector. Let $\mathcal{B}(\lambda)$ denote the crystal basis of $V_{q}(\lambda)$ with $b_{\lambda} \in \mathcal{B}(\lambda)$ the element corresponding to $v_{q, \lambda} \in V_{q}(\lambda)$, and $\tilde{e}_{i}, \tilde{f}_{i}: \mathcal{B}(\lambda) \cup\{0\} \rightarrow \mathcal{B}(\lambda) \cup\{0\}$ for $i \in I$ the Kashiwara operators. Define maps $\varepsilon_{i}, \varphi_{i}: \mathcal{B}(\infty) \rightarrow \mathbb{Z}$ and $\varepsilon_{i}, \varphi_{i}: \mathcal{B}(\lambda) \rightarrow \mathbb{Z}$ for $i \in I$ by

$$
\begin{aligned}
& \varepsilon_{i}(b):=\max \left\{k \in \mathbb{Z}_{\geq 0} \mid \tilde{e}_{i}^{k} b \neq 0\right\}, \varphi_{i}(b):=\varepsilon_{i}(b)+\left\langle\operatorname{wt}(b), h_{i}\right\rangle \text { for } b \in \mathcal{B}(\infty), \text { and } \\
& \varepsilon_{i}(b):=\max \left\{k \in \mathbb{Z}_{\geq 0} \mid \tilde{e}_{i}^{k} b \neq 0\right\}, \varphi_{i}(b):=\max \left\{k \in \mathbb{Z}_{\geq 0} \mid \tilde{f}_{i}^{k} b \neq 0\right\} \text { for } b \in \mathcal{B}(\lambda) .
\end{aligned}
$$

Proposition $2.2\left(\left[17\right.\right.$, Theorem 5]). For $\lambda \in P_{+}$, let $\pi_{\lambda}: U_{q}\left(\mathfrak{u}^{-}\right) \rightarrow V_{q}(\lambda)$ denote the surjective $U_{q}\left(\mathfrak{u}^{-}\right)$module homomorphism given by $u \mapsto u v_{q, \lambda}$.

(1) The homomorphism $\pi_{\lambda}$ induces a surjective map $\mathcal{B}(\infty) \rightarrow \mathcal{B}(\lambda) \cup\{0\}$ (denoted also by $\left.\pi_{\lambda}\right)$. For

$$
\widetilde{\mathcal{B}}(\lambda):=\left\{b \in \mathcal{B}(\infty) \mid \pi_{\lambda}(b) \neq 0\right\},
$$

the restriction map $\pi_{\lambda}: \widetilde{\mathcal{B}}(\lambda) \rightarrow \mathcal{B}(\lambda)$ is bijective.

(2) $\tilde{f}_{i} \pi_{\lambda}(b)=\pi_{\lambda}\left(\tilde{f}_{i} b\right)$ for all $i \in I$ and $b \in \mathcal{B}(\infty)$.

(3) $\tilde{e}_{i} \pi_{\lambda}(b)=\pi_{\lambda}\left(\tilde{e}_{i} b\right)$ for all $i \in I$ and $b \in \widetilde{\mathcal{B}}(\lambda)$.

(4) $\varepsilon_{i}\left(\pi_{\lambda}(b)\right)=\varepsilon_{i}(b)$ and $\varphi_{i}\left(\pi_{\lambda}(b)\right)=\varphi_{i}(b)+\left\langle\lambda, h_{i}\right\rangle$ for all $i \in I$ and $b \in \widetilde{\mathcal{B}}(\lambda)$. 
N. FUJITA

Definition 2.3. Let $\mathbf{i}=\left(i_{1}, \ldots, i_{r}\right) \in I^{r}$ be a reduced word for $w \in W$, and $\lambda \in P_{+}$. By [19, Propositions 3.2.3 and 3.2.5], the subsets

$$
\begin{aligned}
& \mathcal{B}_{w}(\infty):=\left\{\tilde{f}_{i_{1}}^{a_{1}} \cdots \tilde{f}_{i_{r}}^{a_{r}} b_{\infty} \mid a_{1}, \ldots, a_{r} \in \mathbb{Z}_{\geq 0}\right\} \subset \mathcal{B}(\infty) \text { and } \\
& \mathcal{B}_{w}(\lambda):=\left\{\tilde{f}_{i_{1}}^{a_{1}} \cdots \tilde{f}_{i_{r}}^{a_{r}} b_{\lambda} \mid a_{1}, \ldots, a_{r} \in \mathbb{Z}_{\geq 0}\right\} \backslash\{0\} \subset \mathcal{B}(\lambda)
\end{aligned}
$$

are independent of the choice of a reduced word i. These subsets $\mathcal{B}_{w}(\infty), \mathcal{B}_{w}(\lambda)$ are called Demazure crystals.

Proposition 2.4 (see [19, Proposition 3.2.5]). For $\lambda \in P_{+}$and $w \in W$, the equality $\pi_{\lambda}\left(\mathcal{B}_{w}(\infty)\right)=$ $\mathcal{B}_{w}(\lambda) \cup\{0\}$ holds; hence $\pi_{\lambda}$ induces a bijective map $\pi_{\lambda}: \widetilde{\mathcal{B}}_{w}(\lambda) \rightarrow \mathcal{B}_{w}(\lambda)$, where $\widetilde{\mathcal{B}}_{w}(\lambda):=\mathcal{B}_{w}(\infty) \cap \widetilde{\mathcal{B}}(\lambda)$.

In the theory of crystal bases, it is important to give their concrete parametrizations. In this paper, we use two parametrizations: Littelmann's string parametrization and the Kashiwara embedding.

Definition 2.5. Let $\mathbf{i}=\left(i_{1}, \ldots, i_{r}\right) \in I^{r}$ be a reduced word for $w \in W$, and $b \in \mathcal{B}_{w}(\infty)$. Define $\Phi_{\mathbf{i}}(b)=\left(a_{1}, \ldots, a_{r}\right) \in \mathbb{Z}_{\geq 0}^{r}$ by

$$
\begin{aligned}
& a_{1}:=\max \left\{a \in \mathbb{Z}_{\geq 0} \mid \tilde{e}_{i_{1}}^{a} b \neq 0\right\}, \\
& a_{2}:=\max \left\{a \in \mathbb{Z}_{\geq 0} \mid \tilde{e}_{i_{2}}^{a} \tilde{e}_{i_{1}}^{a_{1}} b \neq 0\right\}, \\
& \vdots \\
& a_{r}:=\max \left\{a \in \mathbb{Z}_{\geq 0} \mid \tilde{e}_{i_{r}}^{a} \tilde{e}_{i_{r-1}}^{a_{r-1}} \cdots \tilde{e}_{i_{1}}^{a_{1}} b \neq 0\right\} .
\end{aligned}
$$

The $\Phi_{\mathbf{i}}(b)$ is called Littelmann's string parametrization of $b$ with respect to $\mathbf{i}$ (see [31, Sect. 1]).

By [19, Proposition 3.3.1], we have $\mathcal{B}_{w}(\infty)^{*}=\mathcal{B}_{w^{-1}}(\infty)$; hence the map $\Phi_{\mathbf{i}^{\mathrm{op}}} \circ *: \mathcal{B}_{w}(\infty) \rightarrow \mathbb{Z}_{\geq 0}^{r}$ is well-defined, where $\mathbf{i}^{\text {op }}:=\left(i_{r}, \ldots, i_{1}\right)$ is a reduced word for $w^{-1}$.

Definition 2.6. Let $\mathbf{i}=\left(i_{1}, \ldots, i_{r}\right) \in I^{r}$ be a reduced word for $w \in W$. Define a map $\Psi_{\mathbf{i}}: \mathcal{B}_{w}(\infty) \rightarrow \mathbb{Z}_{\geq 0}^{r}$ by $\Psi_{\mathbf{i}}(b):=\Phi_{\mathbf{i}^{\mathrm{op}}}\left(b^{*}\right)^{\text {op }}$ for $b \in \mathcal{B}_{w}(\infty)$, where $\mathbf{a}^{\mathrm{op}}:=\left(a_{r}, \ldots, a_{1}\right)$ for $\mathbf{a}=\left(a_{1}, \ldots, a_{r}\right) \in \mathbb{Z}_{\geq 0}^{r}$. The map $\Psi_{\mathbf{i}}$ is called the Kashiwara embedding of $\mathcal{B}_{w}(\infty)$ (see [19, Sects. 2 and 3]).

Remark 2.7. By the bijective map $\pi_{\lambda}: \widetilde{\mathcal{B}}_{w}(\lambda) \stackrel{\sim}{\rightarrow} \mathcal{B}_{w}(\lambda)$ in Proposition 2.4 , the maps $\Phi_{\mathbf{i}}$ and $\Psi_{\mathbf{i}}$ can be thought of as ones from $\mathcal{B}_{w}(\lambda)$, called Littelmann's string parametrization of $\mathcal{B}_{w}(\lambda)$ and the Kashiwara embedding of $\mathcal{B}_{w}(\lambda)$, respectively.

Definition 2.8. Let $\mathbf{i}=\left(i_{1}, \ldots, i_{r}\right) \in I^{r}$ be a reduced word for $w \in W$, and $\lambda \in P_{+}$. Define a subset $\mathcal{S}_{\mathbf{i}}^{(\lambda, w)} \subset \mathbb{Z}_{>0} \times \mathbb{Z}^{r}$ by

$$
\mathcal{S}_{\mathbf{i}}^{(\lambda, w)}:=\bigcup_{k>0}\left\{\left(k, \Phi_{\mathbf{i}}(b)\right) \mid b \in \widetilde{\mathcal{B}}_{w}(k \lambda)\right\},
$$

and denote by $\mathcal{C}_{\mathbf{i}}^{(\lambda, w)} \subset \mathbb{R}_{\geq 0} \times \mathbb{R}^{r}$ the smallest real closed cone containing $\mathcal{S}_{\mathbf{i}}^{(\lambda, w)}$. Then, we define a subset $\Delta_{\mathbf{i}}^{(\lambda, w)} \subset \mathbb{R}^{r}$ by

$$
\Delta_{\mathbf{i}}^{(\lambda, w)}:=\left\{\mathbf{a} \in \mathbb{R}^{r} \mid(1, \mathbf{a}) \in \mathcal{C}_{\mathbf{i}}^{(\lambda, w)}\right\}
$$

This subset $\Delta_{\mathbf{i}}^{(\lambda, w)}$ is called Littelmann's string polytope for $\mathcal{B}_{w}(\lambda)$ with respect to $\mathbf{i}$ (see $[21$, Definition 3.5] and [31, Sect. 1]). Also, by replacing $\Phi_{\mathbf{i}}$ with $\Psi_{\mathbf{i}}$ in the definitions of $\mathcal{S}_{\mathbf{i}}^{(\lambda, w)}, \mathcal{C}_{\mathbf{i}}^{(\lambda, w)}$, and $\Delta_{\mathbf{i}}^{(\lambda, w)}$, we obtain $\widetilde{\mathcal{S}}_{\mathbf{i}}^{(\lambda, w)} \subset \mathbb{Z}_{>0} \times \mathbb{Z}^{r}, \widetilde{\mathcal{C}}_{\mathbf{i}}^{(\lambda, w)} \subset \mathbb{R}_{\geq 0} \times \mathbb{R}^{r}$, and $\widetilde{\Delta}_{\mathbf{i}}^{(\lambda, w)} \subset \mathbb{R}^{r}$. We call the subset $\widetilde{\Delta}_{\mathbf{i}}^{(\lambda, w)}$ NakashimaZelevinsky's polytope for $\mathcal{B}_{w}(\lambda)$ with respect to i (see [9, Sect. 2.3], [32, Sects. 3 and 4], [33, Sect. 3.1], and [36, Sect. 3]).

A subset $\mathcal{C} \subset \mathbb{R}_{\geq 0} \times \mathbb{R}^{r}$ is said to be a rational convex polyhedral cone if there exists a finite number of rational points $\mathbf{a}_{1}, \ldots, \mathbf{a}_{l} \in \mathbb{Q}_{\geq 0} \times \mathbb{Q}^{r}$ such that $\mathcal{C}=\mathbb{R}_{\geq 0} \mathbf{a}_{1}+\cdots+\mathbb{R}_{\geq 0} \mathbf{a}_{l}$. A subset $\Delta \subset \mathbb{R}^{r}$ is said to be a rational convex polytope if it is the convex hull of a finite number of rational points.

Proposition 2.9 (see [3, Sect. 3.2 and Theorem 3.10], [9, Corollary 4.3] and [31, Sect. 1]). Let $\mathbf{i}=$ $\left(i_{1}, \ldots, i_{r}\right) \in I^{r}$ be a reduced word for $w \in W$, and $\lambda \in P_{+}$. 
(1) The real closed cones $\mathcal{C}_{\mathbf{i}}^{(\lambda, w)}$ and $\widetilde{\mathcal{C}}_{\mathbf{i}}^{(\lambda, w)}$ are both rational convex polyhedral cones; in addition, the following equalities hold:

$$
\mathcal{S}_{\mathbf{i}}^{(\lambda, w)}=\mathcal{C}_{\mathbf{i}}^{(\lambda, w)} \cap\left(\mathbb{Z}_{>0} \times \mathbb{Z}^{r}\right), \widetilde{\mathcal{S}}_{\mathbf{i}}^{(\lambda, w)}=\widetilde{\mathcal{C}}_{\mathbf{i}}^{(\lambda, w)} \cap\left(\mathbb{Z}_{>0} \times \mathbb{Z}^{r}\right) .
$$

(2) The sets $\Delta_{\mathbf{i}}^{(\lambda, w)}$ and $\widetilde{\Delta}_{\mathbf{i}}^{(\lambda, w)}$ are both rational convex polytopes; in addition, the following equalities hold:

$$
\Phi_{\mathbf{i}}\left(\widetilde{\mathcal{B}}_{w}(\lambda)\right)=\Delta_{\mathbf{i}}^{(\lambda, w)} \cap \mathbb{Z}^{r}, \Psi_{\mathbf{i}}\left(\widetilde{\mathcal{B}}_{w}(\lambda)\right)=\widetilde{\Delta}_{\mathbf{i}}^{(\lambda, w)} \cap \mathbb{Z}^{r}
$$

Remark 2.10. By [3, Theorem 3.10] and [31, Sect. 1], we obtain a system of explicit linear inequalities defining Littelmann's string polytope $\Delta_{i}^{(\lambda, w)}$. In addition, under a certain positivity assumption on $\mathbf{i}$, Nakashima $[32,33]$ gave a system of explicit linear inequalities defining Nakashima-Zelevinsky's polytope $\widetilde{\Delta}_{\mathbf{i}}^{(\lambda, w)}$ (see also $[9$, Corollary 5.3]).

Remark 2.11. In $[9,10]$, the polytope $\widetilde{\Delta}_{\mathbf{i}}^{(\lambda, w)}$ is called Nakashima-Zelevinsky's polyhedral realization. However, the word "polyhedral realization" is originally used in $[32,33,36]$ to mean the realization of a crystal basis as the lattice points in an explicit rational convex polyhedral cone or an explicit rational convex polytope. Hence the terminology in $[9,10]$ is slightly inaccurate.

\section{Perfect bases and Newton-Okounkov polytopes}

In this section, we recall the definition of Newton-Okounkov polytopes of Schubert varieties, following $[12,21,22,23]$.

Let us fix a Borel subgroup $B \subset G$, and denote by $B^{-} \subset G$ the opposite Borel subgroup. Then, the full flag variety is defined to be the quotient space $G / B$. For $w \in W$, let $X(w) \subset G / B$ denote the Schubert variety corresponding to $w$, that is, $X(w)$ is the Zariski closure of $B \widetilde{w} B / B$ in $G / B$, where $\widetilde{w} \in G$ denotes a lift for $w$; note that $X(w)$ is independent of the choice of $\widetilde{w}$. It is well-known that $X(w)$ is a normal projective variety of complex dimension $\ell(w)$; here, $\ell(w)$ denotes the length of $w$. Also, for a given $\lambda \in P_{+}$, we define a line bundle $\mathcal{L}_{\lambda}$ on $G / B$ by

$$
\mathcal{L}_{\lambda}:=(G \times \mathbb{C}) / B,
$$

where $B$ acts on $G \times \mathbb{C}$ on the right as follows:

$$
(g, c) \cdot b=(g b, \lambda(b) c)
$$

for $g \in G, c \in \mathbb{C}$, and $b \in B$. By restricting this bundle, we obtain a line bundle on $X(w)$, which we denote by the same symbol $\mathcal{L}_{\lambda}$. Let $U^{-}$denote the unipotent radical of $B^{-}$with Lie algebra $\mathfrak{u}^{-}$, and regard $U^{-}$as an affine open subvariety of $G / B$ by the following open immersion:

$$
U^{-} \hookrightarrow G / B, u \mapsto u \bmod B
$$

Then we consider the set-theoretic intersection $U^{-} \cap X(w)$ in $G / B$. Since this intersection is an open subset of $X(w)$, it inherits an open subvariety structure from $X(w)$; note that it coincides with the variety structure on $U^{-} \cap X(w)$ as a closed subvariety of $U^{-}$(see [10, Sect. 2]).

Let $\mathfrak{b} \subset \mathfrak{g}$ be the Lie algebra of $B$, and $E_{i}, F_{i}, h_{i} \in \mathfrak{g}, i \in I$, the Chevalley generators such that $\left\{E_{i}, h_{i} \mid i \in I\right\} \subset \mathfrak{b}$ and $\left\{F_{i} \mid i \in I\right\} \subset \mathfrak{u}^{-}$. We set $[k]_{i} !:=[k]_{i}[k-1]_{i} \cdots[1]_{i}$ for $i \in I, k \in \mathbb{Z}_{>0}$, and $[0]_{i} !:=1$, where

$$
[k]_{i}:=\frac{q_{i}^{k}-q_{i}^{-k}}{q_{i}-q_{i}^{-1}} \text { for } i \in I, k \in \mathbb{Z}_{>0} .
$$

Also, let $U_{q, \mathbb{Z}}\left(\mathfrak{u}^{-}\right)$denote the $\mathbb{Z}\left[q^{ \pm 1}\right]$-subalgebra of $U_{q}\left(\mathfrak{u}^{-}\right)$generated by $\left\{f_{i}^{(k)} \mid i \in I, k \in \mathbb{Z}_{\geq 0}\right\}$, where $f_{i}^{(k)}:=f_{i}^{k} /[k]_{i}$ !. Then, the $\mathbb{C}$-algebra $\mathbb{C} \otimes_{\mathbb{Z}\left[q^{ \pm 1}\right]} U_{q, \mathbb{Z}}\left(\mathfrak{u}^{-}\right)$is isomorphic to the universal enveloping algebra $U\left(\mathfrak{u}^{-}\right)$of $\mathfrak{u}^{-}$by $1 \otimes f_{i}^{(k)} \mapsto F_{i}^{k} / k$ !, where the $\mathbb{Z}\left[q^{ \pm 1}\right]$-module structure on $\mathbb{C}$ is given by $q \mapsto 1$; hence this process is called the specialization at $q=1$. We define a $\mathbb{C}$-algebra anti-involution $*$ on $U\left(\mathfrak{u}^{-}\right)$by $F_{i}^{*}:=F_{i}$ for all $i \in I$. The algebra $U\left(\mathfrak{u}^{-}\right)$has a Hopf algebra structure given by the following coproduct $\Delta$, counit $\varepsilon$, and antipode $S$ :

$$
\Delta\left(F_{i}\right)=F_{i} \otimes 1+1 \otimes F_{i}, \varepsilon\left(F_{i}\right)=0, \text { and } S\left(F_{i}\right)=-F_{i}
$$

for $i \in I$. In addition, we regard $U\left(\mathfrak{u}^{-}\right)$as a multigraded $\mathbb{C}$-algebra:

$$
U\left(\mathfrak{u}^{-}\right)=\bigoplus_{\mathbf{d} \in \mathbb{Z}_{\geq 0}^{I}} U\left(\mathfrak{u}^{-}\right)_{\mathbf{d}},
$$


where the homogeneous component $U\left(\mathfrak{u}^{-}\right)_{\mathbf{d}}$ for $\mathbf{d}=\left(d_{i}\right)_{i \in I} \in \mathbb{Z}_{\geq_{0}}^{I}$ is defined to be the $\mathbb{C}$-subspace of $U\left(\mathfrak{u}^{-}\right)$spanned by all those elements $F_{j_{1}} \cdots F_{j_{|\mathbf{d}|}}$ such that the cardinality of $\left\{1 \leq k \leq|\mathbf{d}| \mid j_{k}=i\right\}$ is equal to $d_{i}$ for every $i \in I$; here we set $|\mathbf{d}|:=\sum_{i \in I} d_{i}$. Let

$$
U\left(\mathfrak{u}^{-}\right)_{\mathrm{gr}}^{*}:=\bigoplus_{\mathbf{d} \in \mathbb{Z}_{\geq 0}^{I}} \operatorname{Hom}_{\mathbb{C}}\left(U\left(\mathfrak{u}^{-}\right)_{\mathbf{d}}, \mathbb{C}\right)
$$

be the graded dual of $U\left(\mathfrak{u}^{-}\right)$endowed with the dual Hopf algebra structure. Note that the coordinate ring $\mathbb{C}\left[U^{-}\right]$has a Hopf algebra structure given by the following coproduct $\Delta$, counit $\varepsilon$, and antipode $S$ :

$$
\Delta(f)\left(u_{1}, u_{2}\right)=f\left(u_{1} u_{2}\right), \varepsilon(f)=f(e) \text { and } S(f)(u)=f\left(u^{-1}\right)
$$

for $f \in \mathbb{C}\left[U^{-}\right]$and $u, u_{1}, u_{2} \in U^{-}$, where $e \in U^{-}$denotes the identity element. It is known that this Hopf algebra $\mathbb{C}\left[U^{-}\right]$is isomorphic to the dual Hopf algebra $U\left(\mathfrak{u}^{-}\right)_{\mathrm{gr}}^{*}$ (see, for instance, [11, Proposition $5.1])$.

Definition 3.1 (see [2, Definition 5.30], [14, Definition 2.5], and [15, Sect. 4.2]). A $\mathbb{C}$-basis $\mathbf{B}^{\text {low }} \subset U\left(\mathfrak{u}^{-}\right)$ is said to be (lower) perfect if there exists a bijection $\Xi^{\text {low }}: \mathcal{B}(\infty) \stackrel{\sim}{\longrightarrow} \mathbf{B}^{\text {low }}$ satisfying the following conditions:

(i) $\mathbf{B}^{\text {low }}=\bigcup_{\mathbf{d} \in \mathbb{Z}_{\geq 0}^{I}} \mathbf{B}_{\mathbf{d}}^{\text {low }}$, where $\mathbf{B}_{\mathbf{d}}^{\text {low }}:=\mathbf{B}^{\text {low }} \cap U\left(\mathfrak{u}^{-}\right)_{\mathbf{d}}$ for $\mathbf{d} \in \mathbb{Z}_{\geq 0}^{I}$,

(ii) $\Xi^{\text {low }}\left(b_{\infty}\right)=1$,

(iii) for all $i \in I, b \in \mathcal{B}(\infty)$ and $k \in \mathbb{Z}_{\geq 0}$

$$
F_{i}^{(k)} \cdot \Xi^{\text {low }}(b) \in \mathbb{C}^{\times} \Xi^{\text {low }}\left(\tilde{f}_{i}^{k} b\right)+\sum_{\substack{b^{\prime} \in \mathcal{B}(\infty) ; \operatorname{wt}\left(b^{\prime}\right)=\operatorname{wt}\left(\tilde{f}_{i}^{k} b\right), \varepsilon_{i}\left(b^{\prime}\right)>\varepsilon_{i}\left(\tilde{f}_{i}^{k} b\right)}} \mathbb{C} \Xi^{\text {low }}\left(b^{\prime}\right),
$$

where $\mathbb{C}^{\times}:=\mathbb{C} \backslash\{0\}$.

In addition, we always impose the following $*$-stability condition on a perfect basis:

(iv) $\left(\mathbf{B}^{\text {low }}\right)^{*}=\mathbf{B}^{\text {low }}$.

Proposition 3.2 ([10, Proposition 3.10]). The equality $\Xi^{\text {low }}(b)^{*}=\Xi^{\text {low }}\left(b^{*}\right)$ holds for each $b \in \mathcal{B}(\infty)$.

Example 3.3. Lusztig $[27,28,29]$ and Kashiwara [17] constructed a specific $\mathbb{Z}\left[q^{ \pm 1}\right]$-basis $\left\{G_{q}^{\text {low }}(b) \mid\right.$ $b \in \mathcal{B}(\infty)\}$ of $U_{q, \mathbb{Z}}\left(\mathfrak{u}^{-}\right)$, called the canonical basis or the lower global basis. The specialization $\left\{G^{\text {low }}(b) \mid\right.$ $b \in \mathcal{B}(\infty)\} \subset U\left(\mathfrak{u}^{-}\right)$of $\left\{G_{q}^{\text {low }}(b) \mid b \in \mathcal{B}(\infty)\right\}$ at $q=1$ is a perfect basis by [18, Proposition 5.3.1] and [19, Theorem 2.1.1] (see also [9, Proposition 2.8]).

Example 3.4. When $\mathfrak{g}$ is simply-laced, Lusztig [30] constructed a specific $\mathbb{C}$-basis of $U\left(\mathfrak{u}^{-}\right)$, called the semicanonical basis. This is a perfect basis by [30, Proof of Lemma 2.4 and Sect. 3].

For $\lambda \in P_{+}$, denote by $V(\lambda)$ the irreducible highest weight $\mathfrak{g}$-module with highest weight $\lambda$ with $v_{\lambda} \in$ $V(\lambda)$ the highest weight vector, and by $\pi_{\lambda}: U\left(\mathfrak{u}^{-}\right) \rightarrow V(\lambda)$ the surjective $U\left(\mathfrak{u}^{-}\right)$-module homomorphism given by $u \mapsto u v_{\lambda}$. We set $\Xi_{\lambda}^{\text {low }}\left(\pi_{\lambda}(b)\right):=\pi_{\lambda}\left(\Xi^{\text {low }}(b)\right)$ for $b \in \widetilde{\mathcal{B}}(\lambda)$.

Proposition 3.5 (see [10, Proposition $3.14(1)]$ ). The set $\left\{\Xi_{\lambda}^{\text {low }}(b) \mid b \in \mathcal{B}(\lambda)\right\}$ provides a $\mathbb{C}$-basis of $V(\lambda)$, and the element $\pi_{\lambda}\left(\Xi^{\text {low }}(b)\right)$ is identical to 0 for $b \in \mathcal{B}(\infty) \backslash \widetilde{\mathcal{B}}(\lambda)$.

For $w \in W$, let $v_{w \lambda} \in V(\lambda)$ denote the extremal weight vector of weight $w \lambda$. The Demazure module $V_{w}(\lambda)$ corresponding to $w \in W$ is the $B$-submodule of $V(\lambda)$ given by

$$
V_{w}(\lambda):=\sum_{b \in B} \mathbb{C} b v_{w \lambda} .
$$

By the Borel-Weil type theorem (see [25, Corollary 8.1.26]), we know that the space $H^{0}\left(X(w), \mathcal{L}_{\lambda}\right)$ of global sections is a $B$-module isomorphic to the dual module $V_{w}(\lambda)^{*}:=\operatorname{Hom}_{\mathbb{C}}\left(V_{w}(\lambda), \mathbb{C}\right)$. We consider the following condition (D) for a perfect basis $\mathbf{B}^{\text {low }}=\left\{\Xi^{\text {low }}(b) \mid b \in \mathcal{B}(\infty)\right\}$ (see also Proposition 3.5):

(D) the set $\left\{\Xi_{\lambda}^{\text {low }}(b) \mid b \in \mathcal{B}_{w}(\lambda)\right\}$ is a $\mathbb{C}$-basis of the Demazure module $V_{w}(\lambda)$.

Example 3.6. The specialization $\left\{G^{\text {low }}(b) \mid b \in \mathcal{B}(\infty)\right\}$ of the lower global basis at $q=1$ and the semicanonical basis satisfy condition (D) by [19, Proposition 3.2.3] and [40, Theorem 7.1], respectively. 
Let $\mathbf{B}^{\text {up }}=\left\{\Xi^{\text {up }}(b) \mid b \in \mathcal{B}(\infty)\right\} \subset \mathbb{C}\left[U^{-}\right]=U\left(\mathfrak{u}^{-}\right)_{\mathrm{gr}}^{*}$ be the dual basis of $\mathbf{B}^{\text {low }}=\left\{\Xi^{\text {low }}(b) \mid b \in\right.$ $\mathcal{B}(\infty)\} \subset U\left(\mathfrak{u}^{-}\right)$. Recall that $U^{-} \cap X(w)$ is a Zariski closed subvariety of $U^{-}$. Denote by $\eta_{w}: \mathbb{C}\left[U^{-}\right] \rightarrow$ $\mathbb{C}\left[U^{-} \cap X(w)\right]$ the restriction map, and by $\Xi_{w}^{\text {up }}(b) \in \mathbb{C}\left[U^{-} \cap X(w)\right]$ for $b \in \mathcal{B}(\infty)$ the image of $\Xi^{\text {up }}(b) \in$ $\mathbb{C}\left[U^{-}\right]$under $\eta_{w}$. If $\mathbf{B}^{\text {low }}$ satisfies condition (D), then let $\left\{\Xi_{\lambda, w}^{\text {up }}(b) \mid b \in \mathcal{B}_{w}(\lambda)\right\} \subset H^{0}\left(X(w), \mathcal{L}_{\lambda}\right)=$ $V_{w}(\lambda)^{*}$ denote the dual basis of $\left\{\Xi_{\lambda}^{\text {low }}(b) \mid b \in \mathcal{B}_{w}(\lambda)\right\} \subset V_{w}(\lambda)$, and set $\tau_{\lambda}:=\Xi_{\lambda, w}^{\text {up }}\left(b_{\lambda}\right)$.

Lemma 3.7 (see the proof of $\left[9\right.$, Lemma 4.5]). The section $\tau_{\lambda} \in H^{0}\left(X(w), \mathcal{L}_{\lambda}\right)$ does not vanish on $U^{-} \cap X(w)$. Hence the map $H^{0}\left(X(w), \mathcal{L}_{\lambda}\right) \rightarrow \mathbb{C}\left[U^{-} \cap X(w)\right],\left.\tau \mapsto\left(\tau / \tau_{\lambda}\right)\right|_{\left(U^{-} \cap X(w)\right)}$, is well-defined; this map is also denoted by $\iota_{\lambda}$.

Since $U^{-} \cap X(w)$ is an open subvariety of $X(w)$, we see that the map $\iota_{\lambda}: H^{0}\left(X(w), \mathcal{L}_{\lambda}\right) \rightarrow \mathbb{C}\left[U^{-} \cap\right.$ $X(w)]$ is injective.

Proposition 3.8 ([10, Corollary 3.18]). Let $\mathbf{B}^{\mathrm{up}}=\left\{\Xi^{\mathrm{up}}(b) \mid b \in \mathcal{B}(\infty)\right\} \subset \mathbb{C}\left[U^{-}\right]$be the dual basis of a perfect basis satisfying condition (D).

(1) The following equality holds:

$$
\mathbb{C}\left[U^{-} \cap X(w)\right]=\bigcup_{\lambda \in P_{+}} \iota_{\lambda}\left(H^{0}\left(X(w), \mathcal{L}_{\lambda}\right)\right) .
$$

(2) The element $\Xi_{w}^{\mathrm{up}}(b)$ is identical to $\iota_{\lambda}\left(\Xi_{\lambda, w}^{\mathrm{up}}\left(\pi_{\lambda}(b)\right)\right)$ for every $b \in \widetilde{\mathcal{B}}_{w}(\lambda)$.

(3) The set $\left\{\Xi_{w}^{\mathrm{up}}(b) \mid b \in \mathcal{B}_{w}(\infty)\right\}$ provides a $\mathbb{C}$-basis of $\mathbb{C}\left[U^{-} \cap X(w)\right]$.

(4) The element $\Xi_{w}^{\mathrm{up}}(b)$ is identical to 0 unless $b \in \mathcal{B}_{w}(\infty)$.

Let $\mathbf{i}=\left(i_{1}, \ldots, i_{r}\right) \in I^{r}$ be a reduced word for $w \in W$. It is known that the morphism $\mathbb{C}^{r} \rightarrow$ $U^{-} \cap X(w),\left(t_{1}, \ldots, t_{r}\right) \mapsto \exp \left(t_{1} F_{i_{1}}\right) \cdots \exp \left(t_{r} F_{i_{r}}\right) \bmod B$, is birational. Therefore, the function field $\mathbb{C}(X(w))=\mathbb{C}\left(U^{-} \cap X(w)\right)$ is identified with the rational function field $\mathbb{C}\left(t_{1}, \ldots, t_{r}\right)$.

Definition 3.9. We define two lexicographic orders $<$ and $\prec$ on $\mathbb{Z}^{r}$ as follows: $\left(a_{1}, \ldots, a_{r}\right)<\left(a_{1}^{\prime}, \ldots, a_{r}^{\prime}\right)$ (resp., $\left.\left(a_{1}, \ldots, a_{r}\right) \prec\left(a_{1}^{\prime}, \ldots, a_{r}^{\prime}\right)\right)$ if and only if there exists $1 \leq k \leq r$ such that $a_{1}=a_{1}^{\prime}, \ldots, a_{k-1}=$ $a_{k-1}^{\prime}, a_{k}<a_{k}^{\prime}$ (resp., $a_{r}=a_{r}^{\prime}, \ldots, a_{k+1}=a_{k+1}^{\prime}, a_{k}<a_{k}^{\prime}$ ). The lexicographic order $<$ on $\mathbb{Z}^{r}$ induces a total order (denoted by the same symbol $<$ ) on the set of all monomials in the polynomial ring $\mathbb{C}\left[t_{1}, \ldots, t_{r}\right]$ as follows: $t_{1}^{a_{1}} \cdots t_{r}^{a_{r}}<t_{1}^{a_{1}^{\prime}} \cdots t_{r}^{a_{r}^{\prime}}$ if and only if $\left(a_{1}, \ldots, a_{r}\right)<\left(a_{1}^{\prime}, \ldots, a_{r}^{\prime}\right)$. Let us define a $\operatorname{map} v_{\mathbf{i}}: \mathbb{C}(X(w)) \backslash\{0\} \rightarrow \mathbb{Z}^{r}$ by $v_{\mathbf{i}}(f / g):=v_{\mathbf{i}}(f)-v_{\mathbf{i}}(g)$ for $f, g \in \mathbb{C}\left[t_{1}, \ldots, t_{r}\right] \backslash\{0\}$, and by

$$
v_{\mathbf{i}}(f):=-\left(a_{1}, \ldots, a_{r}\right) \text { for } f=c t_{1}^{a_{1}} \cdots t_{r}^{a_{r}}+(\text { lower terms }) \in \mathbb{C}\left[t_{1}, \ldots, t_{r}\right] \backslash\{0\},
$$

where $c \in \mathbb{C} \backslash\{0\}$, and we mean by "lower terms" a linear combination of monomials smaller than $t_{1}^{a_{1}} \cdots t_{r}^{a_{r}}$ with respect to the total order $<$. Similarly, we define a map $\tilde{v}_{\mathbf{i}}$ by using the lexicographic order $\prec$ on $\mathbb{Z}^{r}$; more precisely, we set

$$
\tilde{v}_{\mathbf{i}}(f):=-\left(a_{1}, \ldots, a_{r}\right) \text { for } f=c t_{1}^{a_{1}} \cdots t_{r}^{a_{r}}+(\text { lower terms }) \in \mathbb{C}\left[t_{1}, \ldots, t_{r}\right] \backslash\{0\},
$$

where $c \in \mathbb{C} \backslash\{0\}$.

The map $v_{\mathbf{i}}$ is a valuation, that is, it satisfies the following conditions:

$$
\begin{aligned}
& v_{\mathbf{i}}(f \cdot g)=v_{\mathbf{i}}(f)+v_{\mathbf{i}}(g), \\
& v_{\mathbf{i}}(c \cdot f)=v_{\mathbf{i}}(f), \\
& v_{\mathbf{i}}(f+g) \geq \min \left\{v_{\mathbf{i}}(f), v_{\mathbf{i}}(g)\right\} \text { with respect to the lexicographic order }<\text { unless } f+g=0
\end{aligned}
$$

for $f, g \in \mathbb{C}(X(w)) \backslash\{0\}$ and $c \in \mathbb{C}$. Similarly, the map $\tilde{v}_{\mathbf{i}}$ is a valuation with respect to the lexicographic order $\prec$.

Example 3.10. If $r=3$ and $f=t_{1} t_{2}+t_{3}^{2} \in \mathbb{C}\left[t_{1}, t_{2}, t_{3}\right]$, then we have $v_{\mathbf{i}}(f)=-(1,1,0)$ and $\tilde{v}_{\mathbf{i}}(f)=$ $-(0,0,2)$.

Definition 3.11. Let $\mathbf{i}=\left(i_{1}, \ldots, i_{r}\right) \in I^{r}$ be a reduced word for $w \in W$, and $\lambda \in P_{+}$. Take $v \in\left\{v_{\mathbf{i}}, \tilde{v}_{\mathbf{i}}\right\}$ and $\tau \in H^{0}\left(X(w), \mathcal{L}_{\lambda}\right) \backslash\{0\}$. We define a subset $S\left(X(w), \mathcal{L}_{\lambda}, v, \tau\right) \subset \mathbb{Z}_{>0} \times \mathbb{Z}^{r}$ by

$$
S\left(X(w), \mathcal{L}_{\lambda}, v, \tau\right):=\bigcup_{k>0}\left\{\left(k, v\left(\sigma / \tau^{k}\right)\right) \mid \sigma \in H^{0}\left(X(w), \mathcal{L}_{\lambda}^{\otimes k}\right) \backslash\{0\}\right\},
$$


and denote by $C\left(X(w), \mathcal{L}_{\lambda}, v, \tau\right) \subset \mathbb{R}_{\geq 0} \times \mathbb{R}^{r}$ the smallest real closed cone containing $S\left(X(w), \mathcal{L}_{\lambda}, v, \tau\right)$. Let us define a subset $\Delta\left(X(w), \mathcal{L}_{\lambda}, v, \tau\right) \subset \mathbb{R}^{r}$ by

$$
\Delta\left(X(w), \mathcal{L}_{\lambda}, v, \tau\right):=\left\{\mathbf{a} \in \mathbb{R}^{r} \mid(1, \mathbf{a}) \in C\left(X(w), \mathcal{L}_{\lambda}, v, \tau\right)\right\}
$$

this is called the Newton-Okounkov polytope of $X(w)$ associated to $\mathcal{L}_{\lambda}, v$, and $\tau$.

We define a linear automorphism $\omega: \mathbb{R} \times \mathbb{R}^{r} \stackrel{\sim}{\rightarrow} \mathbb{R} \times \mathbb{R}^{r}$ by $\omega(k, \mathbf{a}):=(k,-\mathbf{a})$. Recall that $\tau_{\lambda}=$ $\Xi_{\lambda, w}^{\mathrm{up}}\left(b_{\lambda}\right) \in H^{0}\left(X(w), \mathcal{L}_{\lambda}\right)$.

Proposition 3.12 (see [21, Sect. 4]). Let $\mathbf{i}=\left(i_{1}, \ldots, i_{r}\right) \in I^{r}$ be a reduced word for $w \in W, \lambda \in P_{+}$, and $\mathbf{B}^{\text {up }}=\left\{\Xi^{\text {up }}(b) \mid b \in \mathcal{B}(\infty)\right\} \subset \mathbb{C}\left[U^{-}\right]$the dual basis of a perfect basis.

(1) Littelmann's string parametrization $\Phi_{\mathbf{i}}(b)$ is equal to $-v_{\mathbf{i}}\left(\Xi_{w}^{\mathrm{up}}(b)\right)$ for every $b \in \mathcal{B}_{w}(\infty)$.

(2) The following equalities hold:

$$
\begin{aligned}
& \mathcal{S}_{\mathbf{i}}^{(\lambda, w)}=\omega\left(S\left(X(w), \mathcal{L}_{\lambda}, v_{\mathbf{i}}, \tau_{\lambda}\right)\right), \mathcal{C}_{\mathbf{i}}^{(\lambda, w)}=\omega\left(C\left(X(w), \mathcal{L}_{\lambda}, v_{\mathbf{i}}, \tau_{\lambda}\right)\right), \text { and } \\
& \Delta_{\mathbf{i}}^{(\lambda, w)}=-\Delta\left(X(w), \mathcal{L}_{\lambda}, v_{\mathbf{i}}, \tau_{\lambda}\right) .
\end{aligned}
$$

Proposition 3.13 (see [9, Sect. 4]). Let $\mathbf{i}=\left(i_{1}, \ldots, i_{r}\right) \in I^{r}$ be a reduced word for $w \in W, \lambda \in P_{+}$, and $\mathbf{B}^{\text {up }}=\left\{\Xi^{\text {up }}(b) \mid b \in \mathcal{B}(\infty)\right\} \subset \mathbb{C}\left[U^{-}\right]$the dual basis of a perfect basis.

(1) The Kashiwara embedding $\Psi_{\mathbf{i}}(b)$ is equal to $-\tilde{v}_{\mathbf{i}}\left(\Xi_{w}^{\mathrm{up}}(b)\right)$ for every $b \in \mathcal{B}_{w}(\infty)$.

(2) The following equalities hold:

$$
\begin{aligned}
& \widetilde{\mathcal{S}}_{\mathbf{i}}^{(\lambda, w)}=\omega\left(S\left(X(w), \mathcal{L}_{\lambda}, \tilde{v}_{\mathbf{i}}, \tau_{\lambda}\right)\right), \widetilde{\mathcal{C}}_{\mathbf{i}}^{(\lambda, w)}=\omega\left(C\left(X(w), \mathcal{L}_{\lambda}, \tilde{v}_{\mathbf{i}}, \tau_{\lambda}\right)\right), \text { and } \\
& \widetilde{\Delta}_{\mathbf{i}}^{(\lambda, w)}=-\Delta\left(X(w), \mathcal{L}_{\lambda}, \tilde{v}_{\mathbf{i}}, \tau_{\lambda}\right) .
\end{aligned}
$$

Remark 3.14. The author and Oya [10] proved that the valuations $v_{\mathbf{i}}, \tilde{v}_{\mathbf{i}}$ are also identical to ones given by counting the order of zeros along certain sequences of subvarieties of $X(w)$.

\section{Orbit Lie Algebras}

In this section, we apply the folding procedure to crystal bases. First we recall from $[6,7]$ the definition of orbit Lie algebras. Recall that $\mathfrak{g}$ is assumed to be a finite-dimensional simple Lie algebra. We further assume that $\mathfrak{g}$ is of simply-laced type. Denote by $C=\left(c_{i, j}\right)_{i, j \in I}$ the Cartan matrix of $\mathfrak{g}$, where $I$ is an index set for the vertices of the Dynkin diagram. Let $\omega: I \rightarrow I$ be a bijection of order $L$ satisfying $c_{\omega(i), \omega(j)}=c_{i, j}$ for all $i, j \in I$; such a bijection $\omega$ is called a Dynkin diagram automorphism. It induces a Lie algebra automorphism $\omega: \mathfrak{g} \stackrel{\sim}{\longrightarrow} \mathfrak{g}$ of order $L$ defined by:

$$
\omega\left(E_{i}\right)=E_{\omega(i)}, \omega\left(F_{i}\right)=F_{\omega(i)}, \omega\left(h_{i}\right)=h_{\omega(i)}
$$

for $i \in I$; note that the Cartan subalgebra $\mathfrak{t}$ is invariant under $\omega$. Also, we define $\omega^{*}: \mathfrak{t}^{*} \stackrel{\sim}{\longrightarrow} \mathfrak{t}^{*}$ by: $\omega^{*}(\lambda)(h)=\lambda\left(\omega^{-1}(h)\right)$ for $\lambda \in \mathfrak{t}^{*}$ and $h \in \mathfrak{t}$. In this paper, we always impose the following orthogonality condition on $\omega$ :

(O) $c_{i, j}=0$ for all $i \neq j$ in the same $\omega$-orbit.

Let us fix a complete set $\breve{I} \subset I$ of representatives for the $\omega$-orbits in $I$. We set $m_{i}:=\min \left\{k \in \mathbb{Z}_{>0} \mid\right.$ $\left.\omega^{k}(i)=i\right\}$ for $i \in I$, and then set

$$
\breve{c}_{i, j}:=\sum_{0 \leq k<m_{j}} c_{i, \omega^{k}(j)}
$$

for $i, j \in \breve{I}$. Then we can verify that the matrix $\breve{C}:=\left(\breve{c}_{i, j}\right)_{i, j \in \breve{I}}$ is an indecomposable Cartan matrix of finite type (see the list below). The finite-dimensional simple Lie algebra $\breve{\mathfrak{g}}$ with Cartan matrix $\breve{C}$ is called the orbit Lie algebra associated to $\omega$.

Let $U_{q}(\breve{\mathfrak{g}})$ be the quantized enveloping algebra of $\breve{\mathfrak{g}}$ with generators $\breve{e}_{i}, \breve{f}_{i}, \breve{t}_{i}, \breve{t}_{i}^{-1}, i \in \breve{I}$, and $U_{q}\left(\breve{\mathfrak{u}}^{-}\right)$ the $\mathbb{Q}(q)$-subalgebra of $U_{q}(\breve{\mathfrak{g}})$ generated by $\left\{\breve{f}_{i} \mid i \in \breve{I}\right\}$. Denote by $\breve{\mathcal{B}}(\infty)$ the crystal basis of $U_{q}\left(\breve{\mathfrak{u}}^{-}\right)$, by $\breve{b}_{\infty} \in \breve{\mathcal{B}}(\infty)$ the element corresponding to $1 \in U_{q}\left(\breve{\mathfrak{u}}^{-}\right)$, and by $\tilde{e}_{i}, \tilde{f}_{i}: \breve{\mathcal{B}}(\infty) \cup\{0\} \rightarrow \breve{\mathcal{B}}(\infty) \cup\{0\}, i \in \breve{I}$, the Kashiwara operators. Then, the crystal basis $\breve{\mathcal{B}}(\infty)$ is realized as a specific subset of $\mathcal{B}(\infty)$; we recall this realization, following $[34,35,39]$. The Dynkin diagram automorphism $\omega$ induces a $\mathbb{Q}(q)$-algebra automorphism $\omega: U_{q}(\mathfrak{g}) \stackrel{\sim}{\longrightarrow} U_{q}(\mathfrak{g})$ of order $L$ defined by:

$$
\omega\left(e_{i}\right)=e_{\omega(i)}, \omega\left(f_{i}\right)=f_{\omega(i)}, \omega\left(t_{i}\right)=t_{\omega(i)}
$$




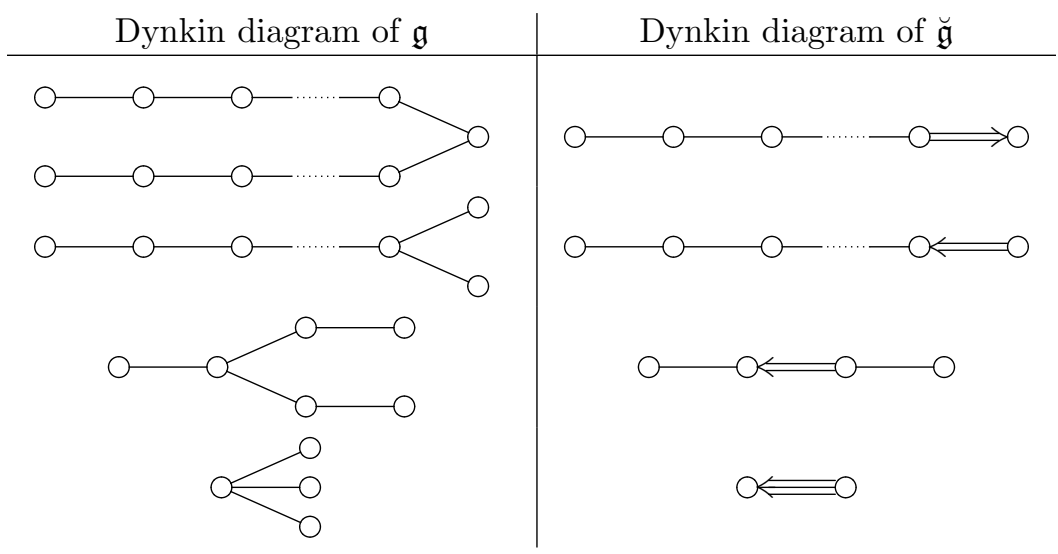

TABLE 1. The list of nontrivial Dynkin diagram automorphisms satisfying assumption (O).

for $i \in I$; remark that $\omega$ preserves $U_{q}\left(\mathfrak{u}^{-}\right)$. We see from [34, Sect. 3.4] that this automorphism induces a natural bijection $\omega: \mathcal{B}(\infty) \rightarrow \mathcal{B}(\infty)$ such that

$$
\omega \circ \tilde{e}_{i}=\tilde{e}_{\omega(i)} \circ \omega \text { and } \omega \circ \tilde{f}_{i}=\tilde{f}_{\omega(i)} \circ \omega
$$

for all $i \in I$. Let us define operators $\tilde{e}_{i}^{\omega}, \tilde{f}_{i}^{\omega}: \mathcal{B}(\infty) \cup\{0\} \rightarrow \mathcal{B}(\infty) \cup\{0\}$ for $i \in I$ by:

$$
\tilde{e}_{i}^{\omega}=\prod_{0 \leq k<m_{i}} \tilde{e}_{\omega^{k}(i)} \text { and } \tilde{f}_{i}^{\omega}=\prod_{0 \leq k<m_{i}} \tilde{f}_{\omega^{k}(i)}
$$

note that the operators $\tilde{e}_{i}, \tilde{e}_{\omega(i)}, \ldots, \tilde{e}_{\omega^{m_{i}-1}(i)}\left(\operatorname{resp} ., \tilde{f}_{i}, \tilde{f}_{\omega(i)}, \ldots, \tilde{f}_{\omega^{m_{i}-1}(i)}\right)$ commute with each other by assumption $(\mathrm{O})$; these operators $\tilde{e}_{i}^{\omega}, \tilde{f}_{i}^{\omega}$ are called the $\omega$-Kashiwara operators. Let $\breve{\mathfrak{t}} \subset \breve{g}$ be a Cartan subalgebra, $\left\{\breve{\alpha}_{i} \in \breve{\mathfrak{t}}^{*} \mid i \in \breve{I}\right\}$ the set of simple roots, $\left\{\breve{h}_{i} \in \breve{\mathfrak{t}} \mid i \in \breve{I}\right\}$ the set of simple coroots, and then set $\mathfrak{t}^{0}:=\{h \in \mathfrak{t} \mid \omega(h)=h\}\left(\mathfrak{t}^{*}\right)^{0}:=\left\{\lambda \in \mathfrak{t}^{*} \mid \omega^{*}(\lambda)=\lambda\right\}$. As in [6, Sect. 2], we obtain $\mathbb{C}$-linear isomorphisms $P_{\omega}: \mathfrak{t}^{0} \stackrel{\sim}{\longrightarrow} \breve{\mathfrak{t}}$ and $P_{\omega}^{*}: \breve{\mathfrak{t}}^{*} \stackrel{\sim}{\longrightarrow}\left(\mathfrak{t}^{0}\right)^{*} \simeq\left(\mathfrak{t}^{*}\right)^{0}$ such that

$$
P_{\omega}^{-1}\left(\breve{h}_{i}\right)=\frac{1}{m_{i}} \sum_{0 \leq k<m_{i}} h_{\omega^{k}(i)}, P_{\omega}^{*}\left(\breve{\alpha}_{i}\right)=\sum_{0 \leq k<m_{i}} \alpha_{\omega^{k}(i)}, \text { and }\left(P_{\omega}^{*}(\breve{\lambda})\right)(h)=\breve{\lambda}\left(P_{\omega}(h)\right)
$$

for $i \in \breve{I}, \breve{\lambda} \in \breve{\mathfrak{t}}^{*}$, and $h \in \mathfrak{t}^{0}$. We denote by $\breve{W}$ the Weyl group of $\breve{\mathfrak{g}}$, and set

$$
\widetilde{W}:=\left\{w \in W \mid \omega^{*} \circ w=w \circ \omega^{*} \text { on } \mathfrak{t}^{*}\right\} .
$$

Then we see from [6, Sect. 3] that there exists a group isomorphism $\Theta: \breve{W} \stackrel{\sim}{\longrightarrow} \widetilde{W}$ such that $\Theta(\breve{w})=$ $P_{\omega}^{*} \circ \breve{w} \circ\left(P_{\omega}^{*}\right)^{-1}$ on $\left(\mathfrak{t}^{*}\right)^{0}$ for all $\breve{w} \in \breve{W}$.

Proposition 4.1 ([34, Theorem 3.4.1]). Let

$$
\mathcal{B}^{0}(\infty):=\{b \in \mathcal{B}(\infty) \mid \omega(b)=b\}
$$

denote the fixed point subset by $\omega$.

(1) The set $\mathcal{B}^{0}(\infty) \cup\{0\}$ is stable under the $\omega$-Kashiwara operators $\tilde{e}_{i}^{\omega}, \tilde{f}_{i}^{\omega}$ for all $i \in I$.

(2) There exists a unique bijective map $P_{\infty}: \mathcal{B}^{0}(\infty) \cup\{0\} \rightarrow \breve{\mathcal{B}}(\infty) \cup\{0\}$ such that

$$
P_{\infty}\left(b_{\infty}\right)=\breve{b}_{\infty}, P_{\infty} \circ \tilde{e}_{i}^{\omega}=\tilde{e}_{i} \circ P_{\infty} \text {, and } P_{\infty} \circ \tilde{f}_{i}^{\omega}=\tilde{f}_{i} \circ P_{\infty}
$$

for all $i \in \breve{I}$.

(3) The equality

$$
P_{\infty}\left(\mathcal{B}_{\Theta(w)}^{0}(\infty)\right)=\breve{\mathcal{B}}_{w}(\infty)
$$

holds for every $w \in \breve{W}$, where $\mathcal{B}_{\Theta(w)}^{0}(\infty):=\mathcal{B}^{0}(\infty) \cap \mathcal{B}_{\Theta(w)}(\infty)$.

For $i \in \breve{I}$ and $b \in \mathcal{B}^{0}(\infty)$, we set

$$
\varepsilon_{i}^{\omega}(b):=\max \left\{a \in \mathbb{Z}_{\geq 0} \mid\left(\tilde{e}_{i}^{\omega}\right)^{a} b \neq 0\right\} .
$$

The properties of $P_{\infty}$ in Proposition 4.1 (2) imply the equality

for every $i \in \breve{I}$ and $b \in \mathcal{B}^{0}(\infty)$.

$$
\varepsilon_{i}^{\omega}(b)=\varepsilon_{i}\left(P_{\infty}(b)\right)
$$


Proposition 4.2. The equality

$$
\varepsilon_{i}^{\omega}(b)=\varepsilon_{\omega^{k}(i)}(b)
$$

holds for every $i \in \breve{I}, k \in \mathbb{Z}_{\geq 0}$, and $b \in \mathcal{B}^{0}(\infty)$.

Proof. Although this is proved in [35, Lemma 2.3.2], we give a proof for the convenience of the reader. By replacing $\breve{I}$ if necessary, we may assume that $k=0$. Since $\left(\tilde{e}_{i}^{\omega}\right)^{a}=\tilde{e}_{\omega^{m_{i}-1}(i)}^{a} \cdots \tilde{e}_{\omega(i)}^{a} \tilde{e}_{i}^{a}$ for $a \in \mathbb{Z}_{\geq 0}$ by assumption $(\mathrm{O})$, the condition $\left(\tilde{e}_{i}^{\omega}\right)^{\varepsilon_{i}^{\omega}(b)} b \neq 0$ implies that $\tilde{e}_{i}^{\varepsilon_{i}^{\omega}(b)} b \neq 0$. Suppose, for a contradiction, that $\tilde{e}_{i}^{\varepsilon_{i}^{\omega}(b)+1} b \neq 0$. Then we have

$$
\begin{array}{rlr}
\tilde{e}_{\omega^{k}(i)}^{\varepsilon_{i}^{\omega}(b)+1} b & =\tilde{e}_{\omega^{k}(i)}^{\varepsilon_{i}^{\omega}(b)+1} \omega^{k}(b) & \left(\text { since } b \in \mathcal{B}^{0}(\infty)\right) \\
& =\omega^{k}\left(\tilde{e}_{i}^{\varepsilon_{i}^{\omega}(b)+1} b\right) & \\
& \neq 0
\end{array}
$$

from which we deduce by assumption $(\mathrm{O})$ that

$$
\tilde{e}_{\omega^{k}(i)}^{\varepsilon_{i}^{\omega}(b)+1} \cdots \tilde{e}_{\omega(i)}^{\varepsilon_{i}^{\omega}(b)+1} \tilde{e}_{i}^{\varepsilon_{i}^{\omega}(b)+1} b \neq 0
$$

for any $0 \leq k \leq m_{i}-1$; this contradicts the equality $\left(\tilde{e}_{i}^{\omega}\right)^{\varepsilon_{i}^{\omega}(b)+1} b=0$. Therefore, the equality $\tilde{e}_{i}^{\varepsilon_{i}^{\omega}(b)+1} b=0$ holds, which implies that $\varepsilon_{i}(b)=\varepsilon_{i}^{\omega}(b)$. This proves the proposition.

Note that $\breve{P}:=\left(P_{\omega}^{*}\right)^{-1}\left(P \cap\left(\mathfrak{t}^{*}\right)^{0}\right) \subset \breve{\mathfrak{t}}^{*}$ is identical to the weight lattice for $\breve{\mathfrak{g}}$. For $\lambda \in P_{+} \cap\left(\mathfrak{t}^{*}\right)^{0}$, we have a natural bijective map $\omega: \mathcal{B}(\lambda) \rightarrow \mathcal{B}(\lambda)$, induced by the $\mathbb{Q}(q)$-algebra automorphism $\omega: U_{q}(\mathfrak{g}) \stackrel{\sim}{\longrightarrow}$ $U_{q}(\mathfrak{g})$, such that

$$
\omega \circ \tilde{e}_{i}=\tilde{e}_{\omega(i)} \circ \omega \text { and } \omega \circ \tilde{f}_{i}=\tilde{f}_{\omega(i)} \circ \omega
$$

for all $i \in I$ (see [34, Sect. 3.2] and [39, Sect. 3]). Here we recall that $\pi_{\lambda}: \mathcal{B}(\infty) \rightarrow \mathcal{B}(\lambda) \cup\{0\}$ is the canonical map induced from the natural surjection $U_{q}\left(\mathfrak{u}^{-}\right) \rightarrow V_{q}(\lambda)$. If we set

$$
\mathcal{B}^{0}(\lambda):=\{b \in \mathcal{B}(\lambda) \mid \omega(b)=b\}
$$

then it is easily checked that $\pi_{\lambda}\left(\mathcal{B}^{0}(\infty)\right)=\mathcal{B}^{0}(\lambda) \cup\{0\}$. For $\breve{\lambda} \in\left(P_{\omega}^{*}\right)^{-1}\left(P_{+} \cap\left(\mathfrak{t}^{*}\right)^{0}\right)$, let $\breve{V}_{q}(\breve{\lambda})$ denote the irreducible highest weight $U_{q}(\breve{\mathfrak{g}})$-module with highest weight $\breve{\lambda}, \breve{\mathcal{B}}(\breve{\lambda})$ the crystal basis of $V_{q}(\breve{\lambda})$ with $b_{\breve{\lambda}} \in \breve{\mathcal{B}}(\breve{\lambda})$ the highest element, and $\tilde{e}_{i}, \tilde{f}_{i}: \breve{\mathcal{B}}(\breve{\lambda}) \cup\{0\} \rightarrow \breve{\mathcal{B}}(\breve{\lambda}) \cup\{0\}, i \in \breve{I}$, the Kashiwara operators.

Proposition 4.3 ([34, Proposition 3.2.1]). Let $\lambda \in P_{+} \cap\left(\mathfrak{t}^{*}\right)^{0}$.

(1) The set $\mathcal{B}^{0}(\lambda) \cup\{0\}$ is stable under the $\omega$-Kashiwara operators $\tilde{e}_{i}^{\omega}, \tilde{f}_{i}^{\omega}: \mathcal{B}(\lambda) \cup\{0\} \rightarrow \mathcal{B}(\lambda) \cup\{0\}$ for all $i \in I$, defined in the same way as $\omega$-Kashiwara operators for $\mathcal{B}(\infty)$.

(2) There exists a unique bijective map $P_{\lambda}: \mathcal{B}^{0}(\lambda) \cup\{0\} \rightarrow \breve{\mathcal{B}}(\breve{\lambda}) \cup\{0\}$ such that

$$
P_{\lambda}\left(b_{\lambda}\right)=b_{\breve{\lambda}}, P_{\lambda} \circ \tilde{e}_{i}^{\omega}=\tilde{e}_{i} \circ P_{\lambda} \text { and } P_{\lambda} \circ \tilde{f}_{i}^{\omega}=\tilde{f}_{i} \circ P_{\lambda}
$$

for all $i \in \breve{I}$, where $\breve{\lambda}:=\left(P_{\omega}^{*}\right)^{-1}(\lambda)$.

(3) The following diagram is commutative:

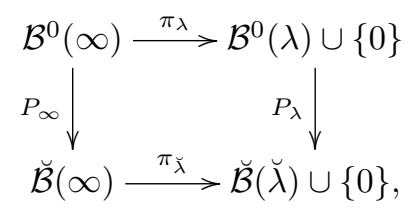

where $\pi_{\breve{\lambda}}$ is the map induced from the natural surjective map $U_{q}\left(\breve{\mathfrak{u}}^{-}\right) \rightarrow \breve{V}_{q}(\breve{\lambda})$.

(4) The equality

$$
P_{\lambda}\left(\mathcal{B}_{\Theta(w)}^{0}(\lambda)\right)=\breve{\mathcal{B}}_{w}(\breve{\lambda})
$$

holds for all $w \in \breve{W}$, where $\mathcal{B}_{\Theta(w)}^{0}(\lambda):=\mathcal{B}^{0}(\lambda) \cap \mathcal{B}_{\Theta(w)}(\lambda)$ and $\breve{\mathcal{B}_{w}}(\breve{\lambda}) \subset \breve{\mathcal{B}}(\breve{\lambda})$ is the corresponding Demazure crystal.

Remark 4.4. The composite maps $\breve{\mathcal{B}}(\infty) \stackrel{P_{\infty}^{-1}}{\longrightarrow} \mathcal{B}^{0}(\infty) \hookrightarrow \mathcal{B}(\infty)$ and $\breve{\mathcal{B}}(\breve{\lambda}) \stackrel{P_{\lambda}^{-1}}{\longrightarrow} \mathcal{B}^{0}(\lambda) \hookrightarrow \mathcal{B}(\lambda)$ are identical to the maps arising from a similarity of crystal bases (see [20, Sect. 5]). This similarity is a variant of what we consider in Section 6 . 
It is easily seen that $\omega \circ *=* \circ \omega$ on $U_{q}(\mathfrak{g})$, which implies the same equality on $\mathcal{B}(\infty)$. Hence it follows that $\mathcal{B}^{0}(\infty)^{*}=\mathcal{B}^{0}(\infty)$. We denote by $*: \breve{\mathcal{B}}(\infty) \rightarrow \breve{\mathcal{B}}(\infty)$ Kashiwara's involution on $\breve{\mathcal{B}}(\infty)$.

Proposition 4.5 ([35, Theorem1]). The following diagram is commutative:

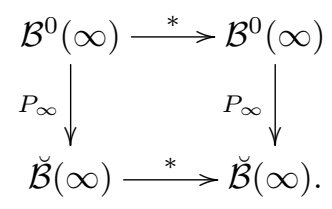

The following is an immediate consequence of Propositions 4.2 and 4.5.

Corollary 4.6. The equality

$$
\varepsilon_{i}\left(P_{\infty}(b)^{*}\right)=\varepsilon_{\omega^{k}(i)}\left(b^{*}\right)
$$

holds for all $i \in \breve{I}, k \in \mathbb{Z}_{\geq 0}$, and $b \in \mathcal{B}^{0}(\infty)$.

Let $\left\{s_{i} \mid i \in I\right\} \subset W$ (resp., $\left\{s_{i} \mid i \in \breve{I}\right\} \subset \breve{W}$ ) be the set of simple reflections. If we take a reduced word $\mathbf{i}=\left(i_{1}, \ldots, i_{r}\right) \in \breve{I}^{r}$ for $w \in \breve{W}$, then we have

$$
\Theta(w)=\Theta\left(s_{i_{1}}\right) \cdots \Theta\left(s_{i_{r}}\right)=s_{i_{1,1}} \cdots s_{i_{1, m_{i_{1}}}} \cdots s_{i_{r, 1}} \cdots s_{i_{r, m_{i_{r}}}},
$$

where we set $i_{k, l}:=\omega^{l-1}\left(i_{k}\right)$ for $1 \leq k \leq r$ and $1 \leq l \leq m_{i_{k}}$. It is easily verified that this is a reduced expression for $\Theta(w)$; we denote by $\Theta(\mathbf{i})$ the corresponding reduced word $\left(i_{1,1}, \ldots, i_{1, m_{i_{1}}}, \ldots, i_{r, 1}, \ldots, i_{r, m_{i_{r}}}\right)$.

Corollary 4.7. Let $\mathbf{i}=\left(i_{1}, \ldots, i_{r}\right) \in \breve{I}^{r}$ be a reduced word for $w \in \breve{W}$. Define an $\mathbb{R}$-linear injective $\operatorname{map} \Upsilon_{\mathbf{i}}: \mathbb{R}^{r} \hookrightarrow \mathbb{R}^{m_{i_{1}}+\cdots+m_{i_{r}}}$ by:

$$
\Upsilon_{\mathbf{i}}\left(a_{1}, \ldots, a_{r}\right)=(\underbrace{a_{1}, \ldots, a_{1}}_{m_{i_{1}}}, \ldots, \underbrace{a_{r}, \ldots, a_{r}}_{m_{i_{r}}}) .
$$

Then, the equalities

$$
\Upsilon_{\mathbf{i}}\left(\Phi_{\mathbf{i}}(b)\right)=\Phi_{\Theta(\mathbf{i})}\left(P_{\infty}^{-1}(b)\right) \text { and } \Upsilon_{\mathbf{i}}\left(\Psi_{\mathbf{i}}(b)\right)=\Psi_{\Theta(\mathbf{i})}\left(P_{\infty}^{-1}(b)\right)
$$

hold for all $b \in \breve{\mathcal{B}}_{w}(\infty)$. In particular, the following equalities hold:

$$
\Upsilon_{\mathbf{i}}\left(\Phi_{\mathbf{i}}\left(\breve{\mathcal{B}}_{w}(\infty)\right)\right)=\Phi_{\Theta(\mathbf{i})}\left(\mathcal{B}_{\Theta(w)}^{0}(\infty)\right) \text {, and } \Upsilon_{\mathbf{i}}\left(\Psi_{\mathbf{i}}\left(\breve{\mathcal{B}}_{w}(\infty)\right)\right)=\Psi_{\Theta(\mathbf{i})}\left(\mathcal{B}_{\Theta(w)}^{0}(\infty)\right) \text {. }
$$

Proof. We take $b \in \breve{\mathcal{B}}_{w}(\infty)$, and write $\Phi_{\mathbf{i}}(b)$ as $\left(a_{1}, \ldots, a_{r}\right)$. We will show that

$$
\Phi_{\Theta(\mathbf{i})}\left(P_{\infty}^{-1}(b)\right)=(\underbrace{a_{1}, \ldots, a_{1}}_{m_{i_{1}}}, \ldots, \underbrace{a_{r}, \ldots, a_{r}}_{m_{i_{r}}}) .
$$

It follows by assumption (O) and Proposition 4.2 that

$$
\varepsilon_{i_{1, k}}\left(\tilde{e}_{i_{1, k-1}}^{a_{1}} \cdots \tilde{e}_{i_{1,1}}^{a_{1}} b\right)=\varepsilon_{i_{1, k}}(b)=a_{1}
$$

for all $1 \leq k \leq m_{i_{1}}$ (see also the proof of Proposition 4.2). Therefore, the following equality holds:

$$
\Phi_{\Theta(\mathbf{i})}\left(P_{\infty}^{-1}(b)\right)=(\underbrace{a_{1}, \ldots, a_{1}}_{m_{i_{1}}}, \Phi_{\Theta\left(\mathbf{i}_{\geq 2}\right)}\left(P_{\infty}^{-1}\left(b^{\prime}\right)\right)),
$$

where $\mathbf{i}_{\geq 2}:=\left(i_{2}, \ldots, i_{r}\right)$ and $b^{\prime}:=\tilde{e}_{i_{1, m_{i_{1}}}}^{a_{1}} \cdots \tilde{e}_{i_{1,1}}^{a_{1}} b$. Moreover, by induction on $r$, we deduce that

$$
\Phi_{\Theta\left(\mathbf{i}_{\geq 2}\right)}\left(P_{\infty}^{-1}\left(b^{\prime}\right)\right)=(\underbrace{a_{2}, \ldots, a_{2}}_{m_{i_{2}}}, \ldots, \underbrace{a_{r}, \ldots, a_{r}}_{m_{i_{r}}}) .
$$

From these, we obtain the assertion for $\Phi_{\mathbf{i}}$. The assertion for $\Psi_{\mathbf{i}}$ is shown similarly by using Corollary 4.6 instead of Proposition 4.2.

If $b \in \mathcal{B}_{\Theta(w)}(\infty)$ satisfies $\Phi_{\Theta(\mathbf{i})}(b)=\Upsilon_{\mathbf{i}}\left(a_{1}, \ldots, a_{r}\right)$ for some $\left(a_{1}, \ldots, a_{r}\right) \in \mathbb{Z}_{\geq 0}^{r}$, then it is easily seen that $b \in \mathcal{B}_{\Theta(w)}^{0}(\infty)$. Hence we obtain the following. 
Corollary 4.8. Let $\mathbf{i}=\left(i_{1}, \ldots, i_{r}\right) \in \breve{I}^{r}$ be a reduced word for $w \in \breve{W}$. Then the following equalities hold:

$$
\begin{aligned}
& \Upsilon_{\mathbf{i}}\left(\Phi_{\mathbf{i}}\left(\breve{\mathcal{B}}_{w}(\infty)\right)\right)=\left\{\left(a_{k, l}\right)_{1 \leq k \leq r, 1 \leq l \leq m_{i_{k}}} \in \Phi_{\Theta(\mathbf{i})}\left(\mathcal{B}_{\Theta(w)}(\infty)\right) \mid a_{k, 1}=\cdots=a_{k, m_{i_{k}}}, 1 \leq k \leq r\right\}, \text { and } \\
& \Upsilon_{\mathbf{i}}\left(\Psi_{\mathbf{i}}\left(\breve{\mathcal{B}}_{w}(\infty)\right)\right)=\left\{\left(a_{k, l}\right)_{1 \leq k \leq r, 1 \leq l \leq m_{i_{k}}} \in \Psi_{\Theta(\mathbf{i})}\left(\mathcal{B}_{\Theta(w)}(\infty)\right) \mid a_{k, 1}=\cdots=a_{k, m_{i_{k}}}, 1 \leq k \leq r\right\}
\end{aligned}
$$

Similarly, we obtain the following (see Proposition $4.3(3),(4))$.

Corollary 4.9. Let $\mathbf{i}=\left(i_{1}, \ldots, i_{r}\right) \in \breve{I}^{r}$ be a reduced word for $w \in \breve{W}$, and $\lambda \in P_{+} \cap\left(\mathfrak{t}^{*}\right)^{0}$. Then the following equalities hold:

$$
\begin{aligned}
& \qquad \Upsilon_{\mathbf{i}}\left(\Phi_{\mathbf{i}}\left(\breve{\mathcal{B}}_{w}(\breve{\lambda})\right)\right)=\left\{\left(a_{k, l}\right)_{1 \leq k \leq r, 1 \leq l \leq m_{i_{k}}} \in \Phi_{\Theta(\mathbf{i})}\left(\mathcal{B}_{\Theta(w)}(\lambda)\right) \mid a_{k, 1}=\cdots=a_{k, m_{i_{k}}}, 1 \leq k \leq r\right\}, \text { and } \\
& \qquad \Upsilon_{\mathbf{i}}\left(\Psi_{\mathbf{i}}\left(\breve{\mathcal{B}}_{w}(\breve{\lambda})\right)\right)=\left\{\left(a_{k, l}\right)_{1 \leq k \leq r, 1 \leq l \leq m_{i_{k}}} \in \Psi_{\Theta(\mathbf{i})}\left(\mathcal{B}_{\Theta(w)}(\lambda)\right) \mid a_{k, 1}=\cdots=a_{k, m_{i_{k}}}, 1 \leq k \leq r\right\}, \\
& \text { where } \breve{\lambda}:=\left(P_{\omega}^{*}\right)^{-1}(\lambda) .
\end{aligned}
$$

By the definitions of Littelmann's string polytopes and Nakashima-Zelevinsky's polytopes, we obtain the following as an immediate consequence of Corollary 4.9.

Corollary 4.10. Let $\mathbf{i}=\left(i_{1}, \ldots, i_{r}\right) \in \breve{I}^{r}$ be a reduced word for $w \in \breve{W}$, and $\lambda \in P_{+} \cap\left(\mathfrak{t}^{*}\right)^{0}$. Then the following equalities hold:

$$
\begin{aligned}
& \Upsilon_{\mathbf{i}}\left(\Delta_{\mathbf{i}}^{(\breve{\lambda}, w)}\right)=\left\{\left(a_{k, l}\right)_{1 \leq k \leq r, 1 \leq l \leq m_{i_{k}}} \in \Delta_{\Theta(\mathbf{i})}^{(\lambda, \Theta(w))} \mid a_{k, 1}=\cdots=a_{k, m_{i_{k}}}, 1 \leq k \leq r\right\}, \text { and } \\
& \Upsilon_{\mathbf{i}}\left(\widetilde{\Delta}_{\mathbf{i}}^{(\breve{\lambda}, w)}\right)=\left\{\left(a_{k, l}\right)_{1 \leq k \leq r, 1 \leq l \leq m_{i_{k}}} \in \widetilde{\Delta}_{\Theta(\mathbf{i})}^{(\lambda, \Theta(w))} \mid a_{k, 1}=\cdots=a_{k, m_{i_{k}}}, 1 \leq k \leq r\right\},
\end{aligned}
$$

where $\breve{\lambda}:=\left(P_{\omega}^{*}\right)^{-1}(\lambda)$.

Remark 4.11. Corollary 4.10 is naturally extended to string polytopes for generalized Demazure modules, defined in [8].

\section{Fixed point Lie subalgebras}

In this section, we prove our main result. Let us consider the fixed point Lie subalgebra by $\omega$

$$
\mathfrak{g}^{\omega}:=\{x \in \mathfrak{g} \mid \omega(x)=x\} .
$$

Define $E_{i}^{\prime}, F_{i}^{\prime}, h_{i}^{\prime} \in \mathfrak{g}^{\omega}$ and $\alpha_{i}^{\prime} \in\left(\mathfrak{t}^{*}\right)^{0}$ for $i \in \breve{I}$ by

$$
E_{i}^{\prime}:=\sum_{0 \leq k<m_{i}} E_{\omega^{k}(i)}, F_{i}^{\prime}:=\sum_{0 \leq k<m_{i}} F_{\omega^{k}(i)}, h_{i}^{\prime}:=\sum_{0 \leq k<m_{i}} h_{\omega^{k}(i)} \text { and } \alpha_{i}^{\prime}:=\frac{1}{m_{i}} \sum_{0 \leq k<m_{i}} \alpha_{\omega^{k}(i)} .
$$

We set $c_{i, j}^{\prime}:=\left\langle\alpha_{j}^{\prime}, h_{i}^{\prime}\right\rangle$ for $i, j \in \breve{I}$. Then, it is easily checked that $\breve{c}_{i, j}=c_{j, i}^{\prime}$ for all $i, j \in \breve{I}$; namely, the matrix $C^{\prime}:=\left(c_{i, j}^{\prime}\right)_{i, j \in \breve{I}}$ is the transpose of $\breve{C}$. In particular, the matrix $C^{\prime}$ is an indecomposable Cartan matrix of finite type.

Proposition 5.1 (see [13, Proposition 8.3]). The fixed point Lie subalgebra $\mathfrak{g}^{\omega}$ is the simple Lie algebra with Cartan matrix $C^{\prime}$ and Chevalley generators $E_{i}^{\prime}, F_{i}^{\prime}, h_{i}^{\prime}, i \in \breve{I}$; in particular, the orbit Lie algebra $\breve{\mathfrak{g}}$ associated to $\omega$ is the (Langlands) dual Lie algebra of $\mathfrak{g}^{\omega}$.

Recall that $G$ is the connected, simply-connected simple algebraic group with $\operatorname{Lie}(G)=\mathfrak{g}$. The Lie algebra automorphism $\omega: \mathfrak{g} \stackrel{\sim}{\longrightarrow} \mathfrak{g}$ induces an algebraic group automorphism $\omega: G \stackrel{\sim}{\rightarrow} G$ such that $\omega(\exp (x))=\exp (\omega(x))$ for all $x \in \mathfrak{g}$. It is known that the fixed point subgroup

$$
G^{\omega}:=\{g \in G \mid \omega(g)=g\}
$$

is a connected simple algebraic group with $\operatorname{Lie}\left(G^{\omega}\right)=\mathfrak{g}^{\omega}$; note that $G^{\omega}$ is a Zariski closed subgroup of $G$. In addition, we see by Table 1 in Section 4 and a case-by-case argument that $G^{\omega}$ is simplyconnected. Since the fixed point subgroup $\left(U^{-}\right)^{\omega}:=U^{-} \cap G^{\omega}$ is a Zariski closed subgroup of $U^{-}$, the coordinate ring $\mathbb{C}\left[\left(U^{-}\right)^{\omega}\right]$ is a quotient of $\mathbb{C}\left[U^{-}\right]$; denote by $\pi^{\omega}: \mathbb{C}\left[U^{-}\right] \rightarrow \mathbb{C}\left[\left(U^{-}\right)^{\omega}\right]$ the quotient map. We set $B^{\omega}:=B \cap G^{\omega}$, and consider the full flag variety $G^{\omega} / B^{\omega}$. Let $\iota^{\omega}: G^{\omega} / B^{\omega} \hookrightarrow G / B$ denote the natural injective map. Since $\omega(B)=B$, the automorphism $\omega: G \stackrel{\sim}{\longrightarrow} G$ induces a variety automorphism $\omega: G / B \stackrel{\sim}{\rightarrow} G / B$, and the image of $\iota^{\omega}$ is identical to the fixed point subvariety $(G / B)^{\omega}$. In addition, 
the map $\iota^{\omega}$ induces a $\mathbb{C}$-linear isomorphism from the tangent space of $G^{\omega} / B^{\omega}$ at $e \bmod B^{\omega}$ to that of $(G / B)^{\omega}$ at $e \bmod B$, where $e \in G^{\omega}(\subset G)$ is the identity element; note that both of these tangent spaces are identified with the Lie subalgebra of $\mathfrak{g}^{\omega}$ generated by $\left\{F_{i}^{\prime} \mid i \in \breve{I}\right\}$. Therefore, the map $\iota^{\omega}: G^{\omega} / B^{\omega} \rightarrow(G / B)^{\omega}$ is an isomorphism of varieties (see, for instance, [41, Sect. 5]). Here we note that since $\mathfrak{g}^{\omega}$ is the (Langlands) dual Lie algebra of $\breve{\mathfrak{g}}$, the Weyl group $\breve{W}$ of $\breve{\mathfrak{g}}$ is identified with that of $\mathfrak{g}^{\omega}$. We consider the Schubert variety $X(w) \subset G^{\omega} / B^{\omega} \simeq(G / B)^{\omega}$ corresponding to $w \in \breve{W}$; this is identified with a Zariski closed subvariety of $X(\Theta(w))$. Let us regard $\left(U^{-}\right)^{\omega}$ as an affine open subvariety of $G^{\omega} / B^{\omega}$, and take the intersection $\left(U^{-}\right)^{\omega} \cap X(w)$ in $G^{\omega} / B^{\omega}$ for $w \in \breve{W}$; this intersection is identified with a Zariski closed subvariety of $U^{-} \cap X(\Theta(w))$. Let $\pi_{w}^{\omega}: \mathbb{C}\left[U^{-} \cap X(\Theta(w))\right] \rightarrow \mathbb{C}\left[\left(U^{-}\right)^{\omega} \cap X(w)\right]$ be the restriction map for $w \in \breve{W}$. We take a reduced word $\mathbf{i}=\left(i_{1}, \ldots, i_{r}\right) \in \breve{I}^{r}$ for $w \in \breve{W}$, and regard the coordinate ring $\mathbb{C}\left[\left(U^{-}\right)^{\omega} \cap X(w)\right]$ as a $\mathbb{C}$-subalgebra of the polynomial ring $\mathbb{C}\left[t_{1}, \ldots, t_{r}\right]$ by the following birational morphism:

$$
\mathbb{C}^{r} \rightarrow\left(U^{-}\right)^{\omega} \cap X(w),\left(t_{1}, \ldots, t_{r}\right) \mapsto \exp \left(t_{1} F_{i_{1}}^{\prime}\right) \cdots \exp \left(t_{r} F_{i_{r}}^{\prime}\right)
$$

Since $\Theta(\mathbf{i})=\left(i_{1,1}, \ldots, i_{1, m_{i_{1}}}, \ldots, i_{r, 1}, \ldots, i_{r, m_{i_{r}}}\right)$ is a reduced word for $\Theta(w) \in W$, the coordinate ring $\mathbb{C}\left[U^{-} \cap X(\Theta(w))\right]$ is regarded as a $\mathbb{C}$-subalgebra of the polynomial ring $\mathbb{C}\left[t_{k, l} \mid 1 \leq k \leq r, 1 \leq l \leq m_{i_{k}}\right]$ by the following birational morphism:

$$
\mathbb{C}^{m_{i_{1}}+\cdots+m_{i_{r}}} \rightarrow U^{-} \cap X(\Theta(w)),\left(t_{1,1}, \ldots, t_{r, m_{i_{r}}}\right) \mapsto \exp \left(t_{1,1} F_{i_{1,1}}\right) \cdots \exp \left(t_{r, m_{i_{r}}} F_{i_{r, m_{i_{r}}}}\right) .
$$

Also, under the inclusion map $\left(U^{-}\right)^{\omega} \cap X(w) \hookrightarrow U^{-} \cap X(\Theta(w))$, we have

$$
\exp \left(t F_{i_{k}}^{\prime}\right) \mapsto \exp \left(t F_{i_{k, 1}}\right) \cdots \exp \left(t F_{i_{k, m_{i}}}\right)
$$

for $t \in \mathbb{C}$ and $1 \leq k \leq r$. Hence we obtain the following.

Lemma 5.2. Define a surjective map $\pi_{\mathbf{i}}^{\omega}: \mathbb{C}\left[t_{k, l} \mid 1 \leq k \leq r, 1 \leq l \leq m_{i_{k}}\right] \rightarrow \mathbb{C}\left[t_{1}, \ldots, t_{r}\right]$ by $\pi_{\mathbf{i}}^{\omega}\left(t_{k, l}\right):=t_{k}$ for $1 \leq k \leq r$ and $1 \leq l \leq m_{i_{k}}$. Then the following diagram is commutative:

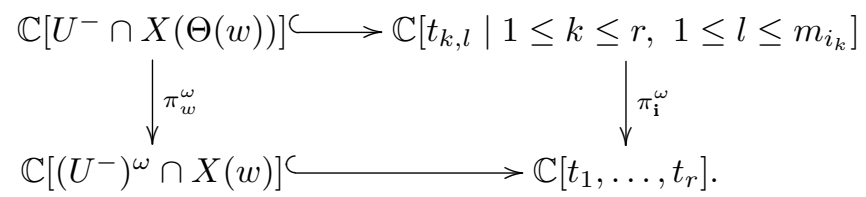

Definition 5.3. Define a $\mathbb{C}$-algebra homomorphism $\Delta: U\left(\mathfrak{u}^{-}\right) \rightarrow U\left(\mathfrak{u}^{-}\right) \otimes U\left(\mathfrak{u}^{-}\right)$by $\Delta(x)=x \otimes 1+1 \otimes x$ for $x \in \mathfrak{u}^{-}$.

Let us consider a perfect basis $\mathbf{B}^{\text {low }}=\left\{\Xi^{\text {low }}(b) \mid b \in \mathcal{B}(\infty)\right\} \subset U\left(\mathfrak{u}^{-}\right)$that satisfies the following positivity conditions:

$(\mathrm{P})_{1}$ the element $F_{i} \cdot \Xi^{\text {low }}(b)$ belongs to $\sum_{b^{\prime} \in \mathcal{B}(\infty)} \mathbb{R}_{\geq 0} \Xi^{\text {low }}\left(b^{\prime}\right)$ for every $b \in \mathcal{B}(\infty)$ and $i \in I$;

$(\mathrm{P})_{2}$ the element $\Delta\left(\Xi^{\text {low }}(b)\right)$ belongs to $\sum_{b^{\prime}, b^{\prime \prime} \in \mathcal{B}(\infty)} \mathbb{R}_{\geq 0} \Xi^{\text {low }}\left(b^{\prime}\right) \otimes \Xi^{\text {low }}\left(b^{\prime \prime}\right)$ for every $b \in \mathcal{B}(\infty)$.

Remark 5.4. In the paper [10], the author and Oya used a perfect basis that satisfies slightly weaker positivity conditions; in it, positivity conditions are imposed only on certain coefficients of $\Delta\left(\Xi^{\text {low }}(b)\right)$.

Example 5.5. Recall that $\mathfrak{g}$ is of simply-laced type. In this case, Lusztig proved that the specialization of the lower global basis at $q=1$ satisfies positivity conditions $(\mathrm{P})_{1},(\mathrm{P})_{2}$ by using the geometric construction of the lower global basis [28, Theorem 11.5].

Lemma 5.6. Let $\mathbf{B}^{\text {low }}=\left\{\Xi^{\text {low }}(b) \mid b \in \mathcal{B}(\infty)\right\} \subset U\left(\mathfrak{u}^{-}\right)$be a perfect basis satisfying $(\mathrm{P})_{1},(\mathrm{P})_{2}$.

(1) The perfect basis $\mathbf{B}^{\text {low }}$ satisfies condition (D) in Section 3.

(2) The element $\Xi_{\Theta(w)}^{\mathrm{up}}(b) \cdot \Xi_{\Theta(w)}^{\mathrm{up}}\left(b^{\prime}\right)$ belongs to $\sum_{b^{\prime \prime} \in \mathcal{B}_{\Theta(w)}(\infty)} \mathbb{R}_{\geq 0} \Xi_{\Theta(w)}^{\mathrm{up}}\left(b^{\prime \prime}\right)$ for all $w \in W$ and $b, b^{\prime} \in \mathcal{B}_{\Theta(w)}(\infty)$; in addition, the coefficient of $\Xi_{\Theta(w)}^{\mathrm{up}}\left(b^{\prime \prime}\right)$ is not equal to 0 if $\Phi_{\Theta(\mathbf{i})}\left(b^{\prime \prime}\right)=$ $\Phi_{\Theta(\mathbf{i})}(b)+\Phi_{\Theta(\mathbf{i})}\left(b^{\prime}\right)$ or if $\Psi_{\Theta(\mathbf{i})}\left(b^{\prime \prime}\right)=\Psi_{\Theta(\mathbf{i})}(b)+\Psi_{\Theta(\mathbf{i})}\left(b^{\prime}\right)$.

(3) The coefficient of $t_{1,1}^{a_{1,1}} \cdots t_{r, m_{i_{r}}}^{a_{r, m_{i_{r}}}}$ in $\Xi_{\Theta(w)}^{\mathrm{up}}(b) \in \mathbb{C}\left[t_{k, l} \mid 1 \leq k \leq r, 1 \leq l \leq m_{i_{k}}\right]$ is a nonnegative real number for all $w \in \breve{W}, b \in \mathcal{B}_{\Theta(w)}(\infty)$, and $a_{1,1}, \ldots, a_{r, m_{i_{r}}} \in \mathbb{Z}_{\geq 0}$. 
Proof. Parts (1), (3), and the first assertion of part (2) are proved in a way similar to [10, Propositions 4.3, 4.7 and Corollary 4.6 (2)]. The second assertion of part (2) follows from general properties of valuations (see [21, Sect. 6]).

Theorem 5.7. Let $\mathbf{i}=\left(i_{1}, \ldots, i_{r}\right) \in \breve{I}^{r}$ be a reduced word for $w \in \breve{W}$, and $\mathbf{B}^{\text {low }}=\left\{\Xi^{\text {low }}(b) \mid b \in\right.$ $\mathcal{B}(\infty)\} \subset U\left(\mathfrak{u}^{-}\right)$a perfect basis satisfying $(\mathrm{P})_{1},(\mathrm{P})_{2}$. Define an $\mathbb{R}$-linear surjective map $\Omega_{\mathbf{i}}: \mathbb{R}^{m_{i_{1}}+\cdots+m_{i_{r}} \rightarrow} \rightarrow$ $\mathbb{R}^{r}$ by:

$$
\Omega_{\mathbf{i}}\left(a_{1,1}, \ldots, a_{1, m_{i_{1}}}, \ldots, a_{r, 1}, \ldots, a_{r, m_{i_{r}}}\right)=\left(a_{1,1}+\cdots+a_{1, m_{i_{1}}}, \ldots, a_{r, 1}+\cdots+a_{r, m_{i_{r}}}\right) .
$$

Then the following equalities hold for all $b \in \mathcal{B}_{\Theta(w)}(\infty)$ :

$$
\begin{aligned}
& v_{\mathbf{i}}\left(\pi_{w}^{\omega}\left(\Xi_{\Theta(w)}^{\text {up }}(b)\right)\right)=\Omega_{\mathbf{i}}\left(v_{\Theta(\mathbf{i})}\left(\Xi_{\Theta(w)}^{\text {up }}(b)\right)\right), \text { and } \\
& \tilde{v}_{\mathbf{i}}\left(\pi_{w}^{\omega}\left(\Xi_{\Theta(w)}^{\text {up }}(b)\right)\right)=\Omega_{\mathbf{i}}\left(\tilde{v}_{\Theta(\mathbf{i})}\left(\Xi_{\Theta(w)}^{\text {up }}(b)\right)\right) .
\end{aligned}
$$

Proof. We prove the assertion only for $v_{\mathbf{i}}$ and $v_{\Theta(\mathbf{i})}$; the proof of the assertion for $\tilde{v}_{\mathbf{i}}$ and $\tilde{v}_{\Theta(\mathbf{i})}$ is similar. We imitate the proof of $\left[10\right.$, Theorem 5.1]. We write $\Phi_{\Theta(\mathbf{i})}(b)=\left(a_{1,1}, \ldots, a_{1, m_{i_{1}}}, \ldots, a_{r, 1}, \ldots, a_{r, m_{i_{r}}}\right)$ for $b \in \mathcal{B}_{\Theta(w)}(\infty)$, and proceed by induction on $r=\ell(w)$ and $a_{1,1}+\cdots+a_{r, m_{i_{r}}}$.

We first consider the case $b \in \mathcal{B}_{s_{i_{1}, 1} \cdots s_{i_{1}, m_{i_{1}}}}(\infty)$, which includes the case $r=1$. In this case, there exist $a_{1}, \ldots, a_{m_{i_{1}}} \in \mathbb{Z}_{\geq 0}$ such that $b=\tilde{f}_{i_{1,1}}^{a_{1}} \ldots \tilde{f}_{i_{1, m_{i_{1}}}}^{a_{m_{i_{1}}}} b_{\infty}$. Then it follows by the definition of $\Phi_{\Theta(\mathbf{i})}$ and assumption $(\mathrm{O})$ in Section 4 that

$$
\begin{aligned}
-v_{\Theta(\mathbf{i})}\left(\Xi_{\Theta(w)}^{\mathrm{up}}(b)\right) & =\Phi_{\Theta(\mathbf{i})}(b) \quad(\text { by Proposition } 3.12(1)) \\
& =\left(a_{1}, \ldots, a_{m_{i_{1}}}, 0, \ldots, 0\right)
\end{aligned}
$$

Hence we deduce by the definition of $v_{\Theta(\mathbf{i})}$ that $\Xi_{\Theta(w)}^{\mathrm{up}}(b)=c t_{1,1}^{a_{1}} \cdots t_{1, m_{i_{1}}}^{a_{m_{i_{1}}}}+$ (other terms) for some $c \in \mathbb{C} \backslash\{0\}$, where "other terms" means a linear combination of monomials of degree $a_{1}+\cdots+a_{m_{i_{1}}}$ that are not equal to $t_{1,1}^{a_{1}} \cdots t_{1, m_{i_{1}}}^{a_{m_{i_{1}}}}$. Here, Lemma 5.6 (3) implies that $c \in \mathbb{R}_{>0}$, and that the coefficients of the "other terms" are also positive real numbers. Therefore, we see from Lemma 5.2 that $\pi_{w}^{\omega}\left(\Xi_{\Theta(w)}^{\text {up }}(b)\right)=$ $c^{\prime} t_{1}^{a_{1}+\cdots+a_{m_{1}}}+$ (other terms) for some $c^{\prime} \in \mathbb{R}_{>0}$, where "other terms" means a linear combination of monomials in $\mathbb{C}\left[t_{1}, \ldots, t_{r}\right]$ of degree $a_{1}+\cdots+a_{m_{i_{1}}}$ that are not equal to $t_{1}^{a_{1}+\cdots+a_{m_{i_{1}}}}$. This implies by the definition of $v_{\mathbf{i}}$ that

$$
\begin{aligned}
v_{\mathbf{i}}\left(\pi_{w}^{\omega}\left(\Xi_{\Theta(w)}^{\mathrm{up}}(b)\right)\right) & =-\left(a_{1}+\cdots+a_{m_{i_{1}}}, 0, \ldots, 0\right) \\
& =\Omega_{\mathbf{i}}\left(v_{\Theta(\mathbf{i})}\left(\Xi_{\Theta(w)}^{\mathrm{up}}(b)\right)\right)
\end{aligned}
$$

We next consider the case $r \geq 2$ and $a_{1,1}=\cdots=a_{1, m_{i_{1}}}=0$. In this case, $b$ is an element of $\mathcal{B}_{\Theta\left(w_{\geq 2}\right)}(\infty)$, where $w_{\geq 2}:=s_{i_{2}} \cdots s_{i_{r}}$. By the definition of $v_{\Theta(\mathbf{i})}$, the equalities $a_{1,1}=\cdots=a_{1, m_{i_{1}}}=0$ imply that $t_{1,1}, \ldots, t_{1, m_{i_{1}}}$ do not appear in $\Xi_{\Theta(w)}^{\mathrm{up}}(b)$, and hence that $t_{1}$ does not appear in $\pi_{w}^{\omega}\left(\Xi_{\Theta(w)}^{\mathrm{up}}(b)\right) \in$ $\mathbb{C}\left[t_{1}, \ldots, t_{r}\right]$. From these, we deduce that

$$
\begin{aligned}
v_{\mathbf{i}}\left(\pi_{w}^{\omega}\left(\Xi_{\Theta(w)}^{\text {up }}(b)\right)\right) & =\left(0, v_{\mathbf{i}_{\geq 2}}\left(\pi_{w_{\geq 2}}^{\omega}\left(\Xi_{\Theta\left(w_{\geq 2}\right)}^{\text {up }}(b)\right)\right)\right) \\
& =\left(0, \Omega_{\mathbf{i}_{\geq 2}}\left(v_{\Theta\left(\mathbf{i}_{\geq 2}\right)}\left(\Xi_{\Theta\left(w_{\geq 2}\right)}^{\text {up }}(b)\right)\right)\right) \quad \text { (by the induction (on } r \text { ) hypothesis) } \\
& =\Omega_{\mathbf{i}}\left(v_{\Theta(\mathbf{i})}\left(\Xi_{\Theta(w)}^{\text {up }}(b)\right)\right),
\end{aligned}
$$

where $\mathbf{i}_{\geq 2}:=\left(i_{2}, \ldots, i_{r}\right)$ is a reduced word for $w_{\geq 2}$.

Finally, consider the case $\left(a_{1,1}, \ldots, a_{1, m_{i_{1}}}\right) \neq(0, \ldots, 0)$ and $b \notin \mathcal{B}_{s_{i_{1}, 1} \cdots s_{i_{1}, m_{i_{1}}}}(\infty)$. We set $b_{1}:=$ $\tilde{f}_{i_{1,1}}^{a_{1,1}} \cdots \tilde{f}_{i_{1, m_{i_{1}}}}^{a_{1, m_{i_{1}}}} b_{\infty}$ and $b_{2}:=\tilde{f}_{i_{2,1}}^{a_{2,1}} \cdots \tilde{f}_{i_{r, m_{i_{r}}}}^{a_{r, m_{i_{r}}}} b_{\infty}$. Then it follows by the definition of $\Phi_{\Theta(\mathbf{i})}$ that $\Phi_{\Theta(\mathbf{i})}\left(b_{1}\right)=$ $\left(a_{1,1}, \ldots, a_{1, m_{i_{1}}}, 0, \ldots, 0\right)$ and $\Phi_{\Theta(\mathbf{i})}\left(b_{2}\right)=\left(0, \ldots, 0, a_{2,1}, \ldots, a_{r, m_{i_{r}}}\right)$; here we have used assumption (O) in Section 4. Hence Proposition 3.12 (1) implies that

$$
\begin{aligned}
v_{\Theta(\mathbf{i})}\left(\Xi_{\Theta(w)}^{\mathrm{up}}(b)\right) & =-\left(a_{1,1}, \ldots, a_{r, m_{i_{r}}}\right) \\
& =-\left(a_{1,1}, \ldots, a_{1, m_{i_{1}}}, 0, \ldots, 0\right)-\left(0, \ldots, 0, a_{2,1}, \ldots, a_{r, m_{i_{r}}}\right) \\
& =v_{\Theta(\mathbf{i})}\left(\Xi_{\Theta(w)}^{\mathrm{up}}\left(b_{1}\right)\right)+v_{\Theta(\mathbf{i})}\left(\Xi_{\Theta(w)}^{\mathrm{up}}\left(b_{2}\right)\right) .
\end{aligned}
$$


Also, we deduce from the induction (on $a_{1,1}+\cdots+a_{r, m_{i_{r}}}$ ) hypothesis that

$$
\begin{aligned}
& \Omega_{\mathbf{i}}\left(v_{\Theta(\mathbf{i})}\left(\Xi_{\Theta(w)}^{\text {up }}\left(b_{1}\right)\right)+v_{\Theta(\mathbf{i})}\left(\Xi_{\Theta(w)}^{\text {up }}\left(b_{2}\right)\right)\right) \\
= & \Omega_{\mathbf{i}}\left(v_{\Theta(\mathbf{i})}\left(\Xi_{\Theta(w)}^{\mathrm{up}}\left(b_{1}\right)\right)\right)+\Omega_{\mathbf{i}}\left(v_{\Theta(\mathbf{i})}\left(\Xi_{\Theta(w)}^{\mathrm{up}}\left(b_{2}\right)\right)\right) \\
= & v_{\mathbf{i}}\left(\pi_{w}^{\omega}\left(\Xi_{\Theta(w)}^{\mathrm{up}}\left(b_{1}\right)\right)\right)+v_{\mathbf{i}}\left(\pi_{w}^{\omega}\left(\Xi_{\Theta(w)}^{\mathrm{up}}\left(b_{2}\right)\right)\right) \\
= & v_{\mathbf{i}}\left(\pi_{w}^{\omega}\left(\Xi_{\Theta(w)}^{\mathrm{up}}\left(b_{1}\right) \cdot \Xi_{\Theta(w)}^{\mathrm{up}}\left(b_{2}\right)\right)\right)
\end{aligned}
$$

(since $v_{\mathbf{i}}$ is a valuation and $\pi_{w}^{\omega}$ is a $\mathbb{C}$-algebra homomorphism).

From these, it follows that

$$
v_{\mathbf{i}}\left(\pi_{w}^{\omega}\left(\Xi_{\Theta(w)}^{\mathrm{up}}\left(b_{1}\right) \cdot \Xi_{\Theta(w)}^{\mathrm{up}}\left(b_{2}\right)\right)\right)=\Omega_{\mathbf{i}}\left(v_{\Theta(\mathbf{i})}\left(\Xi_{\Theta(w)}^{\mathrm{up}}(b)\right)\right) .
$$

Here, by Lemma $5.6(2)$, we have

$$
\Xi_{\Theta(w)}^{\mathrm{up}}\left(b_{1}\right) \cdot \Xi_{\Theta(w)}^{\mathrm{up}}\left(b_{2}\right)=\sum_{b_{3} \in \mathcal{B}_{\Theta(w)}(\infty)} C_{b_{1}, b_{2}}^{\left(b_{3}\right)} \Xi_{\Theta(w)}^{\mathrm{up}}\left(b_{3}\right)
$$

for some $C_{b_{1}, b_{2}}^{\left(b_{3}\right)} \in \mathbb{R}_{\geq 0}, b_{3} \in \mathcal{B}_{\Theta(w)}(\infty)$, with $C_{b_{1}, b_{2}}^{(b)} \neq 0$. By applying $\pi_{w}^{\omega}$ to (5.2), we obtain

$$
\pi_{w}^{\omega}\left(\Xi_{\Theta(w)}^{\mathrm{up}}\left(b_{1}\right) \cdot \Xi_{\Theta(w)}^{\mathrm{up}}\left(b_{2}\right)\right)=\sum_{b_{3} \in \mathcal{B}_{\Theta(w)}(\infty)} C_{b_{1}, b_{2}}^{\left(b_{3}\right)} \pi_{w}^{\omega}\left(\Xi_{\Theta(w)}^{\mathrm{up}}\left(b_{3}\right)\right) .
$$

Since $C_{b_{1}, b_{2}}^{\left(b_{3}\right)} \in \mathbb{R}_{\geq 0}$ for all $b_{3} \in \mathcal{B}_{\Theta(w)}(\infty)$, Lemmas 5.2 and 5.6 (3) imply that no cancellations of monomials occur in the sum on the right-hand side of (5.3). Therefore, we deduce by the definition of $v_{\mathbf{i}}$ that

$$
-v_{\mathbf{i}}\left(\pi_{w}^{\omega}\left(\Xi_{\Theta(w)}^{\mathrm{up}}\left(b_{1}\right) \cdot \Xi_{\Theta(w)}^{\mathrm{up}}\left(b_{2}\right)\right)\right)=\max \left\{-v_{\mathbf{i}}\left(\pi_{w}^{\omega}\left(\Xi_{\Theta(w)}^{\mathrm{up}}\left(b_{3}\right)\right)\right) \mid b_{3} \in \mathcal{B}_{\Theta(w)}(\infty), C_{b_{1}, b_{2}}^{\left(b_{3}\right)} \neq 0\right\},
$$

where "max" means the maximum with respect to the lexicographic order $<$ in Definition 3.9. Since $C_{b_{1}, b_{2}}^{(b)} \neq 0$, we obtain

$$
-v_{\mathbf{i}}\left(\pi_{w}^{\omega}\left(\Xi_{\Theta(w)}^{\mathrm{up}}(b)\right)\right) \leq-v_{\mathbf{i}}\left(\pi_{w}^{\omega}\left(\Xi_{\Theta(w)}^{\mathrm{up}}\left(b_{1}\right) \cdot \Xi_{\Theta(w)}^{\mathrm{up}}\left(b_{2}\right)\right)\right)
$$

Now, by the definition of $v_{\Theta(\mathbf{i})}$ together with the equality $-v_{\Theta(\mathbf{i})}\left(\Xi_{\Theta(w)}^{\mathrm{up}}(b)\right)=\left(a_{1,1}, \ldots, a_{r, m_{i_{r}}}\right)$, the monomial $t_{1,1}^{a_{1,1}} \cdots t_{r, m_{i_{r}}}^{a_{r, m_{i_{r}}}}$ appears in the polynomial $\Xi_{\Theta(w)}^{\mathrm{up}}(b) \in \mathbb{C}\left[t_{1,1}, \ldots, t_{r, m_{i_{r}}}\right]$. Since $C_{b_{1}, b_{2}}^{(b)} \neq 0$ and $C_{b_{1}, b_{2}}^{\left(b_{3}\right)} \in \mathbb{R}_{\geq 0}$ for all $b_{3} \in \mathcal{B}_{\Theta(w)}(\infty)$, we see by Lemmas 5.2 and 5.6 (3) that the monomial

$$
t_{1}^{a_{1,1}+\cdots+a_{1, m_{i}}} \cdots t_{r}^{a_{r, 1}+\cdots+a_{r, m_{i_{r}}}}
$$

appears in the polynomial $\pi_{w}^{\omega}\left(\Xi_{\Theta(w)}^{\mathrm{up}}(b)\right) \in \mathbb{C}\left[t_{1}, \ldots, t_{r}\right]$, which implies that

$$
\begin{aligned}
-\Omega_{\mathbf{i}}\left(v_{\Theta(\mathbf{i})}\left(\Xi_{\Theta(w)}^{\mathrm{up}}(b)\right)\right) & =\left(a_{1,1}+\cdots+a_{1, m_{i_{1}}}, \ldots, a_{r, 1}+\cdots+a_{r, m_{i_{r}}}\right) \\
& \leq-v_{\mathbf{i}}\left(\pi_{w}^{\omega}\left(\Xi_{\Theta(w)}^{\operatorname{up}}(b)\right)\right) .
\end{aligned}
$$

By combining (5.1), (5.4), and (5.5), we conclude that

$$
\Omega_{\mathbf{i}}\left(v_{\Theta(\mathbf{i})}\left(\Xi_{\Theta(w)}^{\mathrm{up}}(b)\right)\right)=v_{\mathbf{i}}\left(\pi_{w}^{\omega}\left(\Xi_{\Theta(w)}^{\mathrm{up}}(b)\right)\right)=v_{\mathbf{i}}\left(\pi_{w}^{\omega}\left(\Xi_{\Theta(w)}^{\mathrm{up}}\left(b_{1}\right) \cdot \Xi_{\Theta(w)}^{\mathrm{up}}\left(b_{2}\right)\right)\right) .
$$

This proves the theorem.

Denote by $P^{\prime} \subset\left(\mathfrak{t}^{*}\right)^{0}$ the subgroup generated by $\varpi_{i}^{\prime}:=\frac{1}{m_{i}} \sum_{0 \leq k<m_{i}} \varpi_{\omega^{k}(i)}, i \in \breve{I}$. Since the set $\left\{h_{i}^{\prime} \mid i \in \breve{I}\right\}$ is regarded as the set of simple coroots of $\mathfrak{g}^{\omega}$, the subgroup $P^{\prime}$ is identified with the weight lattice for $\mathfrak{g}^{\omega}$; in particular, an element $\lambda \in P \cap\left(\mathfrak{t}^{*}\right)^{0}$ gives an integral weight $\hat{\lambda}$ for $\mathfrak{g}^{\omega}$. Recall that for $w \in \breve{W}$, the Schubert variety $X(w) \subset G^{\omega} / B^{\omega} \simeq(G / B)^{\omega}$ is identified with a Zariski closed subvariety of $X(\Theta(w))$. The inclusion map $X(w) \hookrightarrow X(\Theta(w))$ induces a $B^{\omega}$-module homomorphism $H^{0}\left(X(\Theta(w)), \mathcal{L}_{\lambda}\right) \rightarrow H^{0}\left(X(w), \mathcal{L}_{\hat{\lambda}}\right)$ (denoted also by $\left.\pi_{w}^{\omega}\right)$ for $\lambda \in P_{+} \cap\left(\mathfrak{t}^{*}\right)^{0}$. Now we define $\mathbb{C}$-linear injective maps $\iota_{\lambda}: H^{0}\left(X(\Theta(w)), \mathcal{L}_{\lambda}\right) \hookrightarrow \mathbb{C}\left[U^{-} \cap X(\Theta(w))\right]$ and $\iota_{\hat{\lambda}}: H^{0}\left(X(w), \mathcal{L}_{\hat{\lambda}}\right) \hookrightarrow \mathbb{C}\left[\left(U^{-}\right)^{\omega} \cap X(w)\right]$ as in Lemma 3.7. The following is an immediate consequence of the definitions. 
Proposition 5.8. For $\lambda \in P_{+} \cap\left(\mathfrak{t}^{*}\right)^{0}$ and $w \in \breve{W}$, the following diagram is commutative:

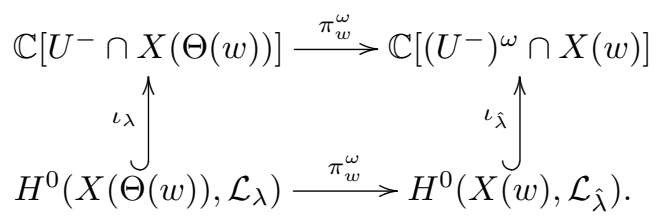

From this, we obtain the following by Propositions 3.8 (2), 5.8, and Theorem 5.7.

Corollary 5.9. The following equalities hold:

$$
\begin{aligned}
& \Omega_{\mathbf{i}}\left(\Delta\left(X(\Theta(w)), \mathcal{L}_{\lambda}, v_{\Theta(\mathbf{i})}, \tau_{\lambda}\right)\right) \subset \Delta\left(X(w), \mathcal{L}_{\hat{\lambda}}, v_{\mathbf{i}}, \tau_{\hat{\lambda}}\right), \text { and } \\
& \Omega_{\mathbf{i}}\left(\Delta\left(X(\Theta(w)), \mathcal{L}_{\lambda}, \tilde{v}_{\Theta(\mathbf{i})}, \tau_{\lambda}\right)\right) \subset \Delta\left(X(w), \mathcal{L}_{\hat{\lambda}}, \tilde{v}_{\mathbf{i}}, \tau_{\hat{\lambda}}\right) .
\end{aligned}
$$

The following is the main result of this paper.

Theorem 5.10. Let $\mathbf{i}=\left(i_{1}, \ldots, i_{r}\right) \in \breve{I}^{r}$ be a reduced word for $w \in \breve{W}$, and $\lambda \in P_{+} \cap\left(\mathfrak{t}^{*}\right)^{0}$. Then the maps

$$
\begin{aligned}
& \Omega_{\mathbf{i}}: \Delta\left(X(\Theta(w)), \mathcal{L}_{\lambda}, v_{\Theta(\mathbf{i})}, \tau_{\lambda}\right) \rightarrow \Delta\left(X(w), \mathcal{L}_{\hat{\lambda}}, v_{\mathbf{i}}, \tau_{\hat{\lambda}}\right) \text { and } \\
& \Omega_{\mathbf{i}}: \Delta\left(X(\Theta(w)), \mathcal{L}_{\lambda}, \tilde{v}_{\Theta(\mathbf{i})}, \tau_{\lambda}\right) \rightarrow \Delta\left(X(w), \mathcal{L}_{\hat{\lambda}}, \tilde{v}_{\mathbf{i}}, \tau_{\hat{\lambda}}\right)
\end{aligned}
$$

are surjective.

In order to prove this theorem, we consider a pair $\left((\mathfrak{g}, \omega: I \rightarrow I),\left(\mathfrak{g}^{\prime}, \omega^{\prime}: I^{\prime} \rightarrow I^{\prime}\right)\right)$ of a simply-laced simple Lie algebra and its Dynkin diagram automorphism. We assume that these satisfy the following conditions:

$(\mathrm{C})_{1}$ the fixed point Lie subalgebra $\left(\mathfrak{g}^{\prime}\right)^{\omega^{\prime}}$ is isomorphic to the orbit Lie algebra $\breve{\mathfrak{g}}$ associated to $\omega$; this condition implies that the index set $\breve{I}$ for $\breve{\mathfrak{g}}$ is identified with the index set $\breve{I}^{\prime}\left(=\left(\breve{I}^{\prime}\right)\right)$ for $\left(\mathfrak{g}^{\prime}\right)^{\omega^{\prime}}$

$(\mathrm{C})_{2}$ if we set $m_{i}:=\min \left\{k \in \mathbb{Z}_{>0} \mid \omega^{k}(i)=i\right\}, i \in \breve{I}$, and $m_{i}^{\prime}:=\min \left\{k \in \mathbb{Z}_{>0} \mid\left(\omega^{\prime}\right)^{k}(i)=i\right\}, i \in \breve{I}^{\prime}$, then the product $m_{i} \cdot m_{i}^{\prime}$ is independent of the choice of $i \in \breve{I} \simeq \breve{I}^{\prime}$.

Remark 5.11. Since the orbit Lie algebra $\breve{\mathfrak{g}}$ associated to $\omega$ is the (Langlands) dual Lie algebra of the fixed point Lie subalgebra $\mathfrak{g}^{\omega}$, a pair $\left((\mathfrak{g}, \omega),\left(\mathfrak{g}^{\prime}, \omega^{\prime}\right)\right)$ satisfies conditions $(\mathrm{C})_{1}$ and $(\mathrm{C})_{2}$ if and only if a pair $\left(\left(\mathfrak{g}^{\prime}, \omega^{\prime}\right),(\mathfrak{g}, \omega)\right)$ satisfies these.

The following three figures give the list of nontrivial pairs satisfying conditions $(\mathrm{C})_{1}$ and $(\mathrm{C})_{2}$ :

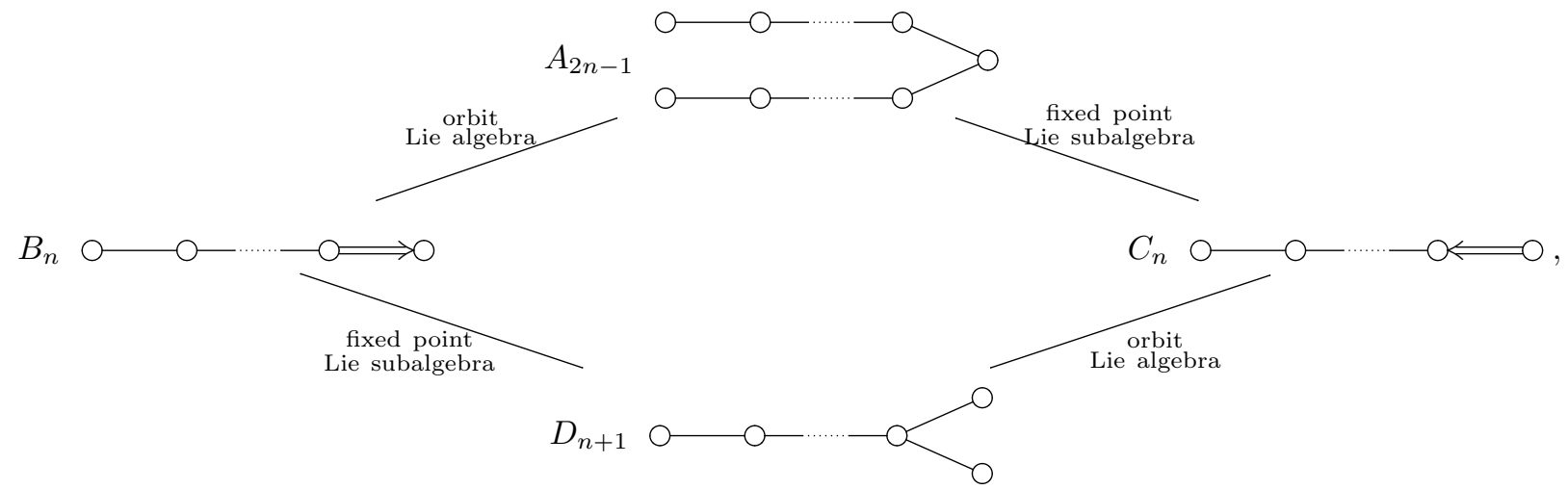




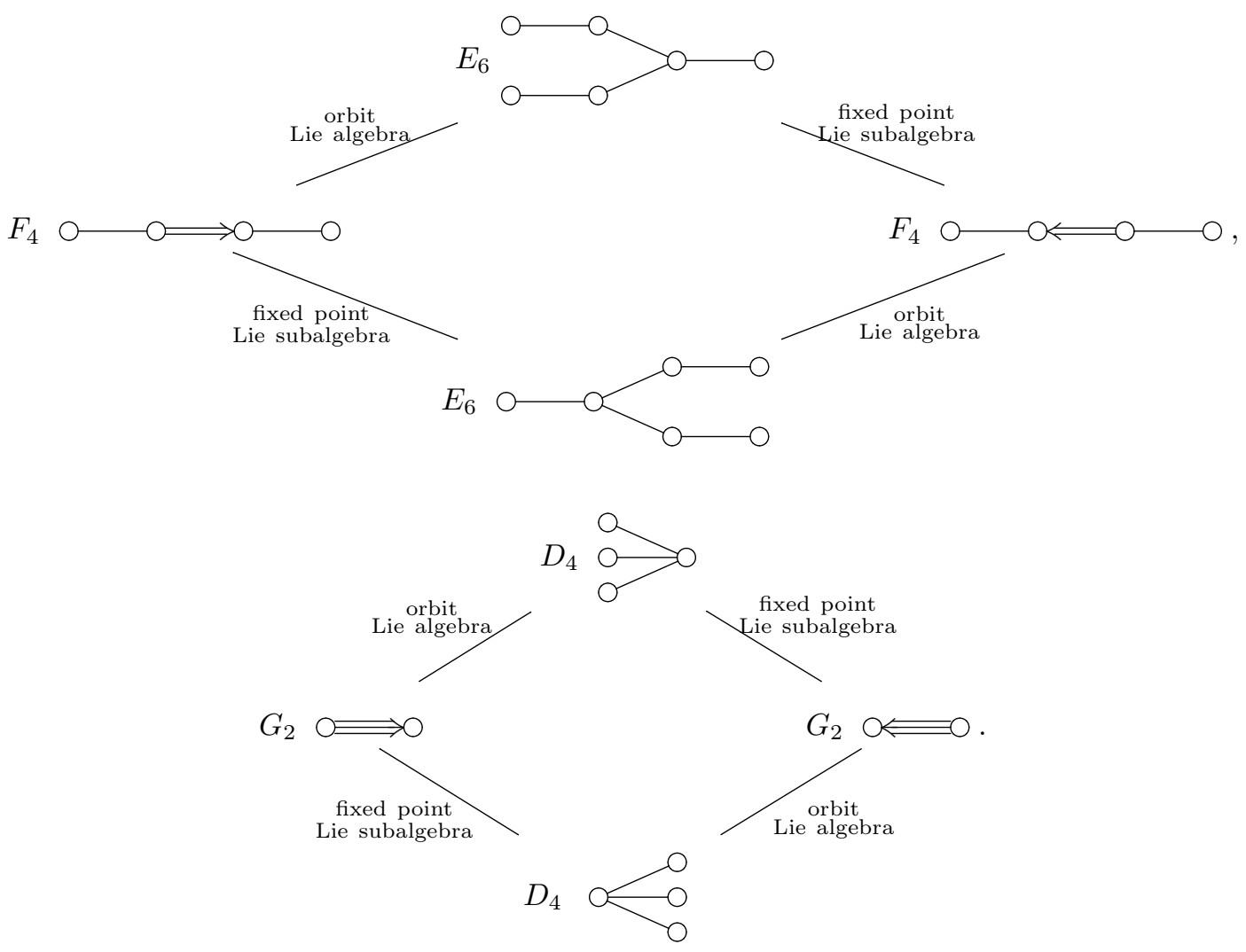

By this list and Table 1 in Section 4, we obtain the following.

Proposition 5.12. For a simply-laced simple Lie algebra $\mathfrak{g}$ with a Dynkin diagram automorphism $\omega$, there exists a simply-laced simple Lie algebra $\mathfrak{g}^{\prime}$ with a Dynkin diagram automorphism $\omega^{\prime}$ such that $\left((\mathfrak{g}, \omega),\left(\mathfrak{g}^{\prime}, \omega^{\prime}\right)\right)$ satisfies conditions $(\mathrm{C})_{1}$ and $(\mathrm{C})_{2}$.

For simplicity, we consider only the pair $\left(A_{2 n-1}, D_{n+1}\right)$; we note that all the arguments below carry over to the other pairs. Denote the Weyl group of type $A_{2 n-1}$ by $W^{A_{2 n-1}}$, the Schubert variety of type $A_{2 n-1}$ by $X^{A_{2 n-1}}(w)$, and so on. We identify $\breve{I}:=\{1, \ldots, n\}$ with the set of vertices of the Dynkin diagram of type $B_{n}$, and also with that of type $C_{n}$ as follows:

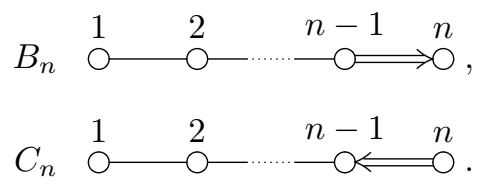

Note that the Weyl group $W^{B_{n}}$ is isomorphic to the Weyl group $W^{C_{n}}$. As we have seen in Section 4 , the Weyl group $W^{B_{n}}\left(\simeq W^{C_{n}}\right)$ is regarded as a specific subgroup of $W^{A_{2 n-1}}$ (resp., of $\left.W^{D_{n+1}}\right)$; let $\Theta: W^{B_{n}} \hookrightarrow W^{A_{2 n-1}}$ (resp., $\Theta^{\prime}: W^{B_{n}} \hookrightarrow W^{D_{n+1}}$ ) be the inclusion map. Take a reduced word $\mathbf{i}=\left(i_{1}, \ldots, i_{r}\right) \in \breve{I}^{r}$ for $w \in W^{B_{n}} \simeq W^{C_{n}}$. The reduced word $\mathbf{i}$ induces a reduced word $\Theta(\mathbf{i})$ (resp., $\Theta^{\prime}(\mathbf{i})$ ) for $\Theta(w)$ (resp., for $\Theta^{\prime}(w)$ ); see Section 4. By Corollary 4.7 and Theorem 5.7, we obtain the following diagrams; we denote the map $\Omega_{\mathbf{i}}: \Phi_{\Theta(\mathbf{i})}\left(\mathcal{B}_{\Theta(w)}^{A_{2 n-1}}(\infty)\right) \rightarrow \Phi_{\mathbf{i}}\left(\mathcal{B}_{w}^{C_{n}}(\infty)\right)$ by $\Omega_{\mathbf{i}}^{A, C}$, the $\operatorname{map} \Upsilon_{\mathbf{i}}: \Phi_{\mathbf{i}}\left(\mathcal{B}_{w}^{B_{n}}(\infty)\right) \rightarrow \Phi_{\Theta(\mathbf{i})}\left(\mathcal{B}_{\Theta(w)}^{A_{2 n-1}}(\infty)\right)$ by $\Upsilon_{\mathbf{i}}^{B, A}$, and so on.

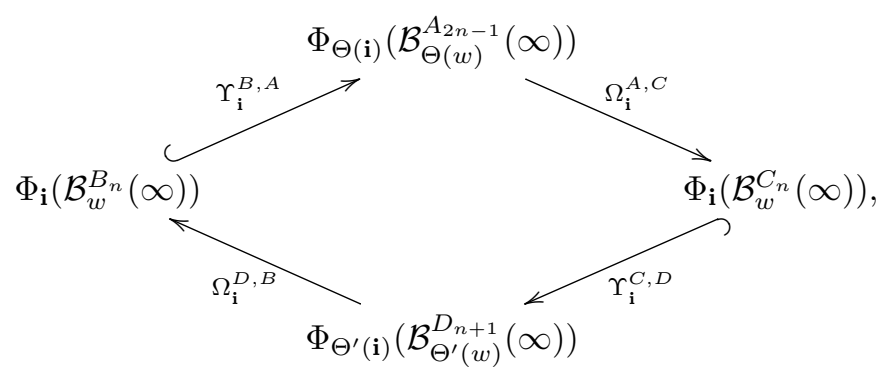




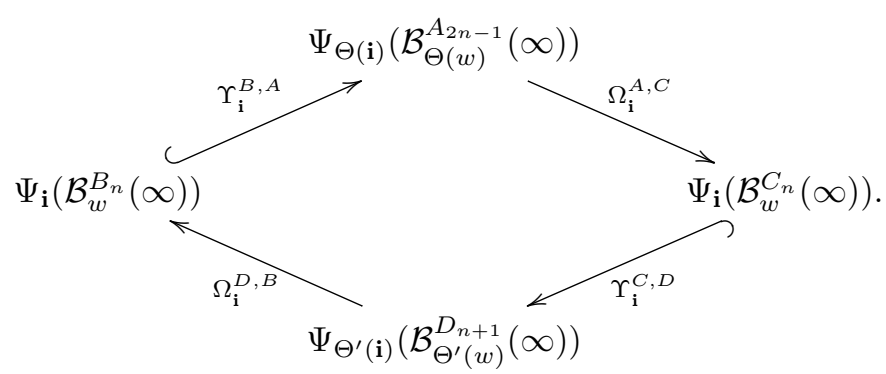

Proof of Theorem 5.10. We give a proof of the assertion only for the map

$$
\Omega_{\mathbf{i}}^{A, C}: \Delta\left(X^{A_{2 n-1}}(\Theta(w)), \mathcal{L}_{\lambda}, v_{\Theta(\mathbf{i})}, \tau_{\lambda}\right) \rightarrow \Delta\left(X^{C_{n}}(w), \mathcal{L}_{\hat{\lambda}}, v_{\mathbf{i}}, \tau_{\hat{\lambda}}\right) ;
$$

the proofs for the other cases are similar. Because

$$
\begin{aligned}
& \Delta\left(X^{A_{2 n-1}}(\Theta(w)), \mathcal{L}_{2 \lambda}, v_{\Theta(\mathbf{i})}, \tau_{2 \lambda}\right)=2 \Delta\left(X^{A_{2 n-1}}(\Theta(w)), \mathcal{L}_{\lambda}, v_{\Theta(\mathbf{i})}, \tau_{\lambda}\right) \text { and } \\
& \Delta\left(X^{C_{n}}(w), \mathcal{L}_{2 \hat{\lambda}}, v_{\mathbf{i}}, \tau_{2 \hat{\lambda}}\right)=2 \Delta\left(X^{C_{n}}(w), \mathcal{L}_{\hat{\lambda}}, v_{\mathbf{i}}, \tau_{\hat{\lambda}}\right),
\end{aligned}
$$

it suffices to prove that the map

$$
\Omega_{\mathbf{i}}^{A, C}: \Delta\left(X^{A_{2 n-1}}(\Theta(w)), \mathcal{L}_{2 \lambda}, v_{\Theta(\mathbf{i})}, \tau_{2 \lambda}\right) \rightarrow \Delta\left(X^{C_{n}}(w), \mathcal{L}_{2 \hat{\lambda}}, v_{\mathbf{i}}, \tau_{2 \hat{\lambda}}\right)
$$

is surjective. By the definitions of $\Omega_{\mathbf{i}}$ and $\Upsilon_{\mathbf{i}}$, we see that $\Omega_{\mathbf{i}}^{A, C} \circ \Upsilon_{\mathbf{i}}^{B, A}\left(a_{1}, \ldots, a_{r}\right)=\left(a_{1}^{\prime}, \ldots, a_{r}^{\prime}\right)$ and $\Omega_{\mathbf{i}}^{D, B} \circ \Upsilon_{\mathbf{i}}^{C, D}\left(a_{1}, \ldots, a_{r}\right)=\left(a_{1}^{\prime \prime}, \ldots, a_{r}^{\prime \prime}\right)$ for $\left(a_{1}, \ldots, a_{r}\right) \in \mathbb{R}^{r}$, where

$$
\begin{aligned}
a_{k}^{\prime} & := \begin{cases}2 a_{k} & \left(i_{k}=1, \ldots, n-1\right), \\
a_{k} & \left(i_{k}=n\right),\end{cases} \\
a_{k}^{\prime \prime} & := \begin{cases}a_{k} & \left(i_{k}=1, \ldots, n-1\right), \\
2 a_{k} & \left(i_{k}=n\right)\end{cases}
\end{aligned}
$$

for $k=1, \ldots, r$. From these, it follows that the composite map $\Omega_{\mathbf{i}}^{A, C} \circ \Upsilon_{\mathbf{i}}^{B, A} \circ \Omega_{\mathbf{i}}^{D, B} \circ \Upsilon_{\mathbf{i}}^{C, D}$ is identical to $2 \cdot \mathrm{id}_{\mathbb{R}^{r}}$. This implies that the map

$$
\Omega_{\mathbf{i}}^{A, C} \circ \Upsilon_{\mathbf{i}}^{B, A} \circ \Omega_{\mathbf{i}}^{D, B} \circ \Upsilon_{\mathbf{i}}^{C, D}: \Delta\left(X^{C_{n}}(w), \mathcal{L}_{\hat{\lambda}}, v_{\mathbf{i}}, \tau_{\hat{\lambda}}\right) \rightarrow \Delta\left(X^{C_{n}}(w), \mathcal{L}_{2 \hat{\lambda}}, v_{\mathbf{i}}, \tau_{2 \hat{\lambda}}\right)
$$

doubles each of the coordinates, and hence is surjective. Therefore, the map (5.6) is also surjective. This proves the theorem.

Example 5.13. Consider the case $n=2$ :

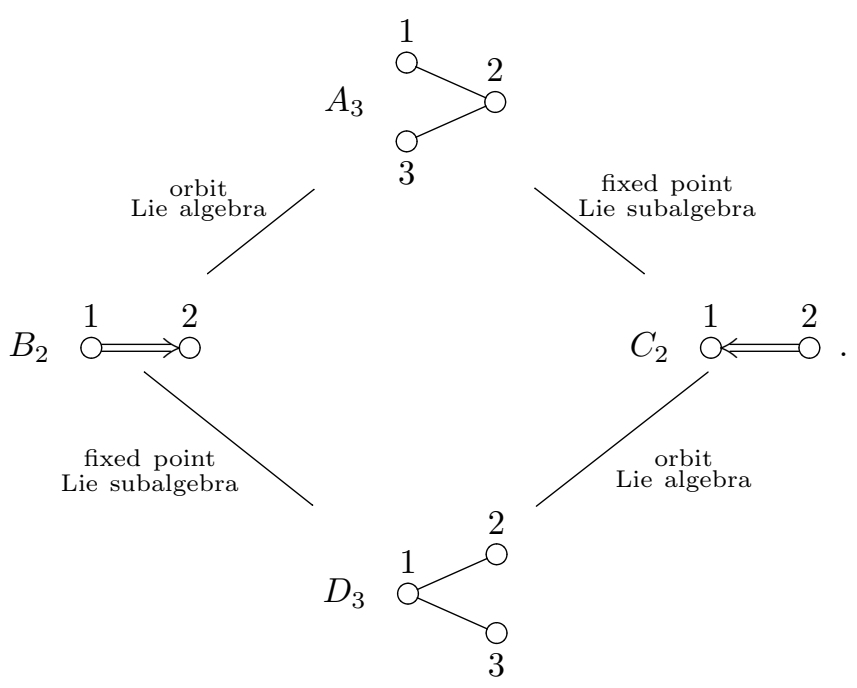


Set $\mathbf{i}:=(1,2,1) \in \breve{I}^{3}$; this is a reduced word for $w:=s_{1} s_{2} s_{1} \in W^{B_{2}} \simeq W^{C_{2}}$. By the definitions of $\Theta$ and $\Theta^{\prime}$, we have $\Theta(\mathbf{i})=(1,3,2,1,3)$ and $\Theta^{\prime}(\mathbf{i})=(1,2,3,1)$. Then, it follows from [31, Sect. 1] that

$$
\begin{aligned}
& \Phi_{\Theta(\mathbf{i})}\left(\mathcal{B}_{\Theta(w)}^{A_{3}}(\infty)\right)=\left\{\left(a_{1}, \ldots, a_{5}\right) \in \mathbb{Z}_{\geq 0}^{5} \mid a_{3} \geq a_{4}, a_{3} \geq a_{5}\right\}, \\
& \Phi_{\mathbf{i}}\left(\mathcal{B}_{w}^{B_{2}}(\infty)\right)=\left\{\left(a_{1}, a_{2}, a_{3}\right) \in \mathbb{Z}_{\geq 0}^{3} \mid a_{2} \geq a_{3}\right\}, \\
& \Phi_{\mathbf{i}}\left(\mathcal{B}_{w}^{C_{2}}(\infty)\right)=\left\{\left(a_{1}, a_{2}, a_{3}\right) \in \mathbb{Z}_{\geq 0}^{3} \mid 2 a_{2} \geq a_{3}\right\}, \\
& \Phi_{\Theta^{\prime}(\mathbf{i})}\left(\mathcal{B}_{\Theta^{\prime}(w)}^{D_{3}}(\infty)\right)=\left\{\left(a_{1}, \ldots, a_{4}\right) \in \mathbb{Z}_{\geq 0}^{4} \mid a_{2}+a_{3} \geq a_{4}\right\} .
\end{aligned}
$$

In addition, the maps $\Omega_{\mathbf{i}}^{A, C}: \mathbb{R}^{5} \rightarrow \mathbb{R}^{3}, \Upsilon_{\mathbf{i}}^{B, A}: \mathbb{R}^{3} \hookrightarrow \mathbb{R}^{5}, \Omega_{\mathbf{i}}^{D, B}: \mathbb{R}^{4} \rightarrow \mathbb{R}^{3}$, and $\Upsilon_{\mathbf{i}}^{C, D}: \mathbb{R}^{3} \hookrightarrow \mathbb{R}^{4}$ are given by

$$
\begin{aligned}
& \Omega_{\mathbf{i}}^{A, C}\left(a_{1}, \ldots, a_{5}\right):=\left(a_{1}+a_{2}, a_{3}, a_{4}+a_{5}\right), \Upsilon_{\mathbf{i}}^{B, A}\left(a_{1}, a_{2}, a_{3}\right):=\left(a_{1}, a_{1}, a_{2}, a_{3}, a_{3}\right), \\
& \Omega_{\mathbf{i}}^{D, B}\left(a_{1}, \ldots, a_{4}\right):=\left(a_{1}, a_{2}+a_{3}, a_{4}\right), \Upsilon_{\mathbf{i}}^{C, D}\left(a_{1}, a_{2}, a_{3}\right):=\left(a_{1}, a_{2}, a_{2}, a_{3}\right) .
\end{aligned}
$$

Through the map $\Omega_{\mathbf{i}}^{A, C}$, the conditions $a_{3} \geq a_{4}, a_{3} \geq a_{5}$ for $\Phi_{\Theta(\mathbf{i})}\left(\mathcal{B}_{\Theta(w)}^{A_{3}}(\infty)\right)$ correspond to the condition $2 a_{2} \geq a_{3}$ for $\Phi_{\mathbf{i}}\left(\mathcal{B}_{w}^{C_{2}}(\infty)\right)$; hence we see that $\Omega_{\mathbf{i}}^{A, C}\left(\Phi_{\Theta(\mathbf{i})}\left(\mathcal{B}_{\Theta(w)}^{A_{3}}(\infty)\right)\right)=\Phi_{\mathbf{i}}\left(\mathcal{B}_{w}^{C_{2}}(\infty)\right)$. Similarly, we observe that the following equalities hold:

$$
\begin{aligned}
& \Omega_{\mathbf{i}}^{D, B}\left(\Phi_{\Theta^{\prime}(\mathbf{i})}\left(\mathcal{B}_{\Theta^{\prime}(w)}^{D_{3}}(\infty)\right)\right)=\Phi_{\mathbf{i}}\left(\mathcal{B}_{w}^{B_{2}}(\infty)\right), \\
& \Upsilon_{\mathbf{i}}^{B, A}\left(\Phi_{\mathbf{i}}\left(\mathcal{B}_{w}^{B_{2}}(\infty)\right)\right)=\left\{\left(a_{1}, \ldots, a_{5}\right) \in \Phi_{\Theta(\mathbf{i})}\left(\mathcal{B}_{\Theta(w)}^{A_{3}}(\infty)\right) \mid a_{1}=a_{2}, a_{4}=a_{5}\right\}, \\
& \Upsilon_{\mathbf{i}}^{C, D}\left(\Phi_{\mathbf{i}}\left(\mathcal{B}_{w}^{C_{2}}(\infty)\right)\right)=\left\{\left(a_{1}, \ldots, a_{4}\right) \in \Phi_{\Theta^{\prime}(\mathbf{i})}\left(\mathcal{B}_{\Theta^{\prime}(w)}^{D_{3}}(\infty)\right) \mid a_{2}=a_{3}\right\} .
\end{aligned}
$$

Take $\lambda \in P_{+}^{A_{3}} \cap\left(\mathfrak{t}^{*}\right)^{0}$ and set $\lambda_{i}:=\left\langle\lambda, h_{i}^{A_{3}}\right\rangle$ for $i=1,2,3$. The condition $\lambda \in\left(\mathfrak{t}^{*}\right)^{0}$ implies that $\lambda_{1}=\lambda_{3}$. By the definition of $\hat{\lambda}$, it follows that $\left\langle\hat{\lambda}, h_{1}^{C_{2}}\right\rangle=2 \lambda_{1}=2 \lambda_{3}$ and $\left\langle\hat{\lambda}, h_{2}^{C_{2}}\right\rangle=\lambda_{2}$. Therefore, we see from Proposition 3.12 (2) and [31, Sect. 1] that $-\Delta\left(X^{A_{2 n-1}}(\Theta(w)), \mathcal{L}_{\lambda}, v_{\Theta(\mathbf{i})}, \tau_{\lambda}\right)$ (resp., $\left.-\Delta\left(X^{C_{n}}(w), \mathcal{L}_{\hat{\lambda}}, v_{\mathbf{i}}, \tau_{\hat{\lambda}}\right)\right)$ is given by the following conditions:

$$
\begin{gathered}
\left(a_{1}, \ldots, a_{5}\right) \in \mathbb{R}_{\geq 0}^{5}, a_{3} \geq a_{4}, a_{3} \geq a_{5}, a_{5} \leq \lambda_{1}, a_{4} \leq \lambda_{1}, \\
a_{3} \leq \lambda_{2}+a_{4}+a_{5}, a_{2} \leq \lambda_{1}+a_{3}-2 a_{5}, a_{1} \leq \lambda_{1}+a_{3}-2 a_{4} \\
\text { (resp., } \left.\left(a_{1}, a_{2}, a_{3}\right) \in \mathbb{R}_{\geq 0}^{3}, 2 a_{2} \geq a_{3}, a_{3} \leq 2 \lambda_{1}, a_{2} \leq \lambda_{2}+a_{3}, a_{1} \leq 2 \lambda_{1}+2 a_{2}-2 a_{3}\right) .
\end{gathered}
$$

Hence it follows that

$$
\Omega_{\mathbf{i}}^{A, C}\left(\Delta\left(X^{A_{2 n-1}}(\Theta(w)), \mathcal{L}_{\lambda}, v_{\Theta(\mathbf{i})}, \tau_{\lambda}\right)\right)=\Delta\left(X^{C_{n}}(w), \mathcal{L}_{\hat{\lambda}}, v_{\mathbf{i}}, \tau_{\hat{\lambda}}\right) .
$$

\section{Relation With SIMILARITy OF CRYSTAL BASES}

In this section, we study the relation of the folding procedure discussed in Sections 4,5 with a similarity of crystal bases.

First we review (a variant of) a similarity property of crystal bases, following [20, Sect. 5]. Let $\mathfrak{g}, I, P,\left\{\alpha_{i}, h_{i} \mid i \in I\right\}$ be as in Section 2, and take $m_{i} \in \mathbb{Z}_{>0}$ for every $i \in I$. We set $\tilde{\alpha}_{i}:=m_{i} \alpha_{i}$, $\tilde{h}_{i}:=\frac{1}{m_{i}} h_{i}$ for $i \in I$, and denote by $\widetilde{P} \subset P$ the set of those $\lambda \in P$ such that $\left\langle\lambda, \tilde{h}_{i}\right\rangle \in \mathbb{Z}$ for all $i \in I$. We impose the following condition on $\left\{m_{i} \mid i \in I\right\}$ :

$$
\tilde{\alpha}_{i} \in \widetilde{P} \text { for all } i \in I \text {. }
$$

Then, it is easily seen that the matrix $\left(\left\langle\tilde{\alpha}_{j}, \tilde{h}_{i}\right\rangle\right)_{i, j \in I}$ is an indecomposable Cartan matrix of finite type. Let $\mathfrak{g}^{\prime}$ be the corresponding simple Lie algebra. Note that the set $\widetilde{P}$ is identified with the weight lattice for $\mathfrak{g}^{\prime}$. Let us write $\mathcal{B}(\infty)$ for $\mathfrak{g}$ as $\mathcal{B}^{\mathfrak{g}}(\infty), \mathcal{B}(\lambda)$ for $\mathfrak{g}$ as $\mathcal{B}^{\mathfrak{g}}(\lambda)$, and so on.

Proposition 6.1 (see the proof of [20, Theorem 5.1]). There exists a unique map $S_{\infty}: \mathcal{B}^{\mathfrak{g}^{\prime}}(\infty) \rightarrow \mathcal{B}^{\mathfrak{g}}(\infty)$ satisfying the following conditions:

(i) $S_{\infty}\left(b_{\infty}^{\mathfrak{g}^{\prime}}\right)=b_{\infty}^{\mathfrak{g}}$,

(ii) $S_{\infty}\left(\tilde{X}_{i} b\right)=\tilde{X}_{i}^{m_{i}} S_{\infty}(b)$ for all $i \in I, b \in \mathcal{B}^{\mathfrak{g}^{\prime}}(\infty)$, and $X \in\{e, f\}$, where $S_{\infty}(0):=0$.

If $\mathfrak{g}$ is of type $B_{n}$ and $\left(m_{1}, \ldots, m_{n-1}, m_{n}\right)=(1, \ldots, 1,2)$, then $\mathfrak{g}^{\prime}$ is the simple Lie algebra of type $C_{n}$. Conversely, if $\mathfrak{g}$ is of type $C_{n}$ and $\left(m_{1}, \ldots, m_{n-1}, m_{n}\right)=(2, \ldots, 2,1)$, then $\mathfrak{g}^{\prime}$ is the simple Lie algebra of type $B_{n}$. Hence we obtain the following.

Corollary 6.2. The following hold. 
(1) There exists a unique map $S_{\infty}^{B, C}: \mathcal{B}^{B_{n}}(\infty) \rightarrow \mathcal{B}^{C_{n}}(\infty)$ satisfying the following conditions:

(i) $S_{\infty}^{B, C}\left(b_{\infty}^{B_{n}}\right)=b_{\infty}^{C_{n}}$,

(ii) $S_{\infty}^{B, C}\left(\tilde{X}_{i} b\right)= \begin{cases}\widetilde{X}_{i}^{2} S_{\infty}^{B, C}(b) & (i=1, \ldots, n-1), \\ \widetilde{X}_{n} S_{\infty}^{B, C}(b) & (i=n)\end{cases}$

for all $b \in \mathcal{B}^{B_{n}}(\infty)$ and $X \in\{e, f\}$, where $S_{\infty}^{B, C}(0):=0$.

(2) There exists a unique map $S_{\infty}^{C, B}: \mathcal{B}^{C_{n}}(\infty) \rightarrow \mathcal{B}^{B_{n}}(\infty)$ satisfying the following conditions:

(i) $S_{\infty}^{C, B}\left(b_{\infty}^{C_{n}}\right)=b_{\infty}^{B_{n}}$,

(ii) $S_{\infty}^{C, B}\left(\tilde{X}_{i} b\right)= \begin{cases}\widetilde{X}_{i} S_{\infty}^{C, B}(b) & (i=1, \ldots, n-1), \\ \widetilde{X}_{n}^{2} S_{\infty}^{C, B}(b) & (i=n)\end{cases}$

for all $b \in \mathcal{B}^{C_{n}}(\infty)$ and $X \in\{e, f\}$, where $S_{\infty}^{C, B}(0):=0$.

It is easily seen that the composite map $S_{\infty}^{C, B} \circ S_{\infty}^{B, C}$ is identical to the map $S_{2}^{B}: \mathcal{B}^{B_{n}}(\infty) \rightarrow \mathcal{B}^{B_{n}}(\infty)$ given by the following conditions:

(i) $S_{2}^{B}\left(b_{\infty}^{B_{n}}\right)=b_{\infty}^{B_{n}}$

(ii) $S_{2}^{B}\left(\tilde{X}_{i} b\right)=\tilde{X}_{i}^{2} S_{2}^{B}(b)$ for all $i \in I, b \in \mathcal{B}^{B_{n}}(\infty)$, and $X \in\{e, f\}$, where $S_{2}^{B}(0):=0$,

(iii) $\varepsilon_{i}\left(S_{2}^{B}(b)\right)=2 \varepsilon_{i}(b)$ and $\varphi_{i}\left(S_{2}^{B}(b)\right)=2 \varphi_{i}(b)$ for all $i \in I$ and $b \in \mathcal{B}^{B_{n}}(\infty)$;

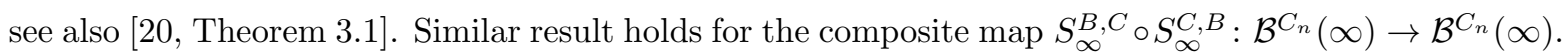
Recall that the Weyl group of type $B_{n}$ is isomorphic to that of type $C_{n}$. By conditions (i) and (ii) in Corollary 6.2 (1) (resp., (2)), it follows that

$$
S_{\infty}^{B, C}\left(\mathcal{B}_{w}^{B_{n}}(\infty)\right) \subset \mathcal{B}_{w}^{C_{n}}(\infty)\left(\text { resp., } S_{\infty}^{C, B}\left(\mathcal{B}_{w}^{C_{n}}(\infty)\right) \subset \mathcal{B}_{w}^{B_{n}}(\infty)\right)
$$

for all $w \in W^{B_{n}} \simeq W^{C_{n}}$.

Proposition 6.3. Let $\mathbf{i}=\left(i_{1}, \ldots, i_{r}\right) \in I^{r}$ be a reduced word for $w \in W^{B_{n}} \simeq W^{C_{n}}$. Then the following equalities hold for all $b \in \mathcal{B}_{w}^{B_{n}}(\infty)$ and $b^{\prime} \in \mathcal{B}_{w}^{C_{n}}(\infty)$ :

$$
\begin{aligned}
& \Phi_{\mathbf{i}}\left(S_{\infty}^{B, C}(b)\right)=\Omega_{\mathbf{i}}^{A, C} \circ \Upsilon_{\mathbf{i}}^{B, A}\left(\Phi_{\mathbf{i}}(b)\right), \Phi_{\mathbf{i}}\left(S_{\infty}^{C, B}\left(b^{\prime}\right)\right)=\Omega_{\mathbf{i}}^{D, B} \circ \Upsilon_{\mathbf{i}}^{C, D}\left(\Phi_{\mathbf{i}}\left(b^{\prime}\right)\right), \text { and } \\
& \Psi_{\mathbf{i}}\left(S_{\infty}^{B, C}(b)\right)=\Omega_{\mathbf{i}}^{A, C} \circ \Upsilon_{\mathbf{i}}^{B, A}\left(\Psi_{\mathbf{i}}(b)\right), \Psi_{\mathbf{i}}\left(S_{\infty}^{C, B}\left(b^{\prime}\right)\right)=\Omega_{\mathbf{i}}^{D, B} \circ \Upsilon_{\mathbf{i}}^{C, D}\left(\Psi_{\mathbf{i}}\left(b^{\prime}\right)\right) .
\end{aligned}
$$

Proof. We prove the assertion only for $S_{\infty}^{B, C}$; the proof of the assertion for $S_{\infty}^{C, B}$ is similar. By equation (5.7) in the proof of Theorem 5.10, it suffices to prove that

$$
\begin{aligned}
& \varepsilon_{i}\left(S_{\infty}^{B, C}(b)\right)= \begin{cases}2 \varepsilon_{i}(b) & (i=1, \ldots, n-1), \\
\varepsilon_{i}(b) & (i=n),\end{cases} \\
& \varepsilon_{i}\left(S_{\infty}^{B, C}(b)^{*}\right)= \begin{cases}2 \varepsilon_{i}\left(b^{*}\right) & (i=1, \ldots, n-1), \\
\varepsilon_{i}\left(b^{*}\right) & (i=n)\end{cases}
\end{aligned}
$$

for all $b \in \mathcal{B}^{B_{n}}(\infty)$. The assertion for $\varepsilon_{i}\left(S_{\infty}^{B, C}(b)^{*}\right)$ follows immediately from the proof of $[20$, Theorem 5.1]. We will prove the assertion for $\varepsilon_{i}\left(S_{\infty}^{B, C}(b)\right)$. If $i=n$, then this is obvious by condition (ii) in Corollary 6.2 (1). For $i=1, \ldots, n-1$, we see by condition (ii) in Corollary 6.2 (1) that

$$
\tilde{e}_{i}^{2 \varepsilon_{i}(b)} S_{\infty}^{B, C}(b)=S_{\infty}^{B, C}\left(\tilde{e}_{i}^{\varepsilon_{i}(b)} b\right) \neq 0 .
$$

Suppose, for a contradiction, that $\tilde{e}_{i}^{2 \varepsilon_{i}(b)+1} S_{\infty}^{B, C}(b) \neq 0$. Then we have

$$
\begin{aligned}
\tilde{e}_{i}^{2 \varepsilon_{i}(b)+1} S_{2}^{B}(b) & =\tilde{e}_{i}^{2 \varepsilon_{i}(b)+1} S_{\infty}^{C, B} \circ S_{\infty}^{B, C}(b) \\
& \left.=S_{\infty}^{C, B}\left(\tilde{e}_{i}^{2 \varepsilon_{i}(b)+1} S_{\infty}^{B, C}(b)\right) \quad \text { (by condition (ii) in Corollary } 6.2(2)\right) \\
& \neq 0,
\end{aligned}
$$

which contradicts condition (iii) for $S_{2}^{B}$ above. Therefore, the equality $\tilde{e}_{i}^{2 \varepsilon_{i}(b)+1} S_{\infty}^{B, C}(b)=0$ holds. From these, we deduce that $\varepsilon_{i}\left(S_{\infty}^{B, C}(b)\right)=2 \varepsilon_{i}(b)$. This proves the proposition.

Remark 6.4. Proposition 6.3 is naturally extended to an arbitrary pair $\left((\mathfrak{g}, \omega),\left(\mathfrak{g}^{\prime}, \omega^{\prime}\right)\right)$ satisfying conditions $(\mathrm{C})_{1}$ and $(\mathrm{C})_{2}$ in Section 5 . 


\section{Appendix A. Case of affine Lie Algebras}

Our arguments in this paper are naturally extended to symmetrizable Kac-Moody algebras. The following figures give the list of nontrivial pairs of automorphisms of simply-laced affine Dynkin diagrams satisfying conditions $(\mathrm{C})_{1}$ and $(\mathrm{C})_{2}$ in Section 5 ; we have used Kac's notation.
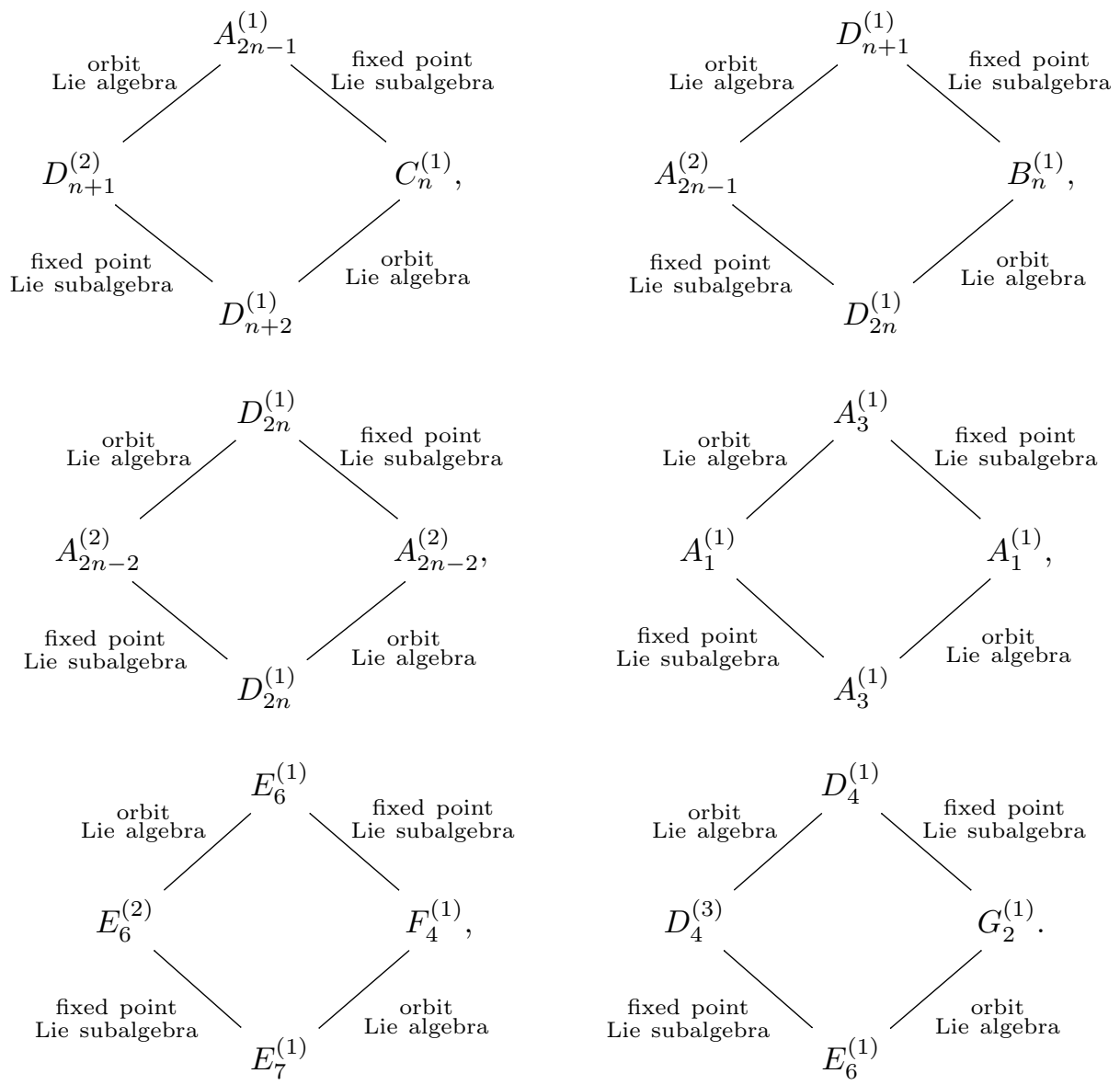

\section{REFERENCES}

[1] D. Anderson, Okounkov bodies and toric degenerations, Math. Ann. 356 (2013), 1183-1202.

[2] A. Berenstein and D. Kazhdan, Geometric and unipotent crystals II: From unipotent bicrystals to crystal bases, in Quantum Groups, Contemp. Math. Vol. 433, Amer. Math. Soc., Providence, RI, 2007, 13-88.

[3] A. Berenstein and A. Zelevinsky, Tensor product multiplicities, canonical bases and totally positive varieties, Invent. Math. 143 (2001), 77-128.

[4] X. Fang, G. Fourier, and P. Littelmann, Essential bases and toric degenerations arising from birational sequences, preprint 2015, arXiv:1510.02295v2.

[5] E. Feigin, G. Fourier, and P. Littelmann, Favourable modules: filtrations, polytopes, Newton-Okounkov bodies and flat degenerations, preprint 2013, arXiv:1306.1292v5, to appear in Transform. Groups.

[6] J. Fuchs, U. Ray, and C. Schweigert, Some automorphisms of generalized Kac-Moody algebras, J. Algebra 191 (1997), 518-540.

[7] J. Fuchs, B. Schellekens, and C. Schweigert, From Dynkin diagram symmetries to fixed point structures, Comm. Math. Phys. 180 (1996), 39-97.

[8] N. Fujita, Newton-Okounkov bodies for Bott-Samelson varieties and string polytopes for generalized Demazure modules, preprint 2015, arXiv:1503.08916v2.

[9] N. Fujita and S. Naito, Newton-Okounkov convex bodies of Schubert varieties and polyhedral realizations of crystal bases, Math. Z. 285 (2017), 325-352.

[10] N. Fujita and H. Oya, A comparison of Newton-Okounkov polytopes of Schubert varieties, preprint 2016, arXiv:1610.08783v1.

[11] C. Geiss, B. Leclerc, and J. Schröer, Preprojective algebras and cluster algebras, in Trends in Representation Theory of Algebras and Related Topics, EMS Ser. Congr. Rep., Eur. Math. Soc., Zürich (2008), 253-283.

[12] M. Harada and K. Kaveh, Integrable systems, toric degenerations, and Okounkov bodies, Invent. Math. 202 (2015), 927-985.

[13] V. G. Kac, Infinite-Dimensional Lie Algebras, 3rd ed., Cambridge University Press, Cambridge, 1990.

[14] S. J. Kang, S. J. Oh, and E. Park, Perfect bases for integrable modules over generalized Kac-Moody algebras, Algebr. Represent. Theory 14 (2011), 571-587. 
[15] S. J. Kang, S. J. Oh, and E. Park, Categorification of quantum generalized Kac-Moody algebras and crystal bases, Internat. J. Math. 23 (2012), 1250116.

[16] M. Kashiwara, Crystallizing the $q$-analogue of universal enveloping algebras, Comm. Math. Phys. 133 (1990), 249-260.

[17] M. Kashiwara, On crystal bases of the $q$-analogue of universal enveloping algebras, Duke Math. J. 63 (1991), 465-516.

[18] M. Kashiwara, Global crystal bases of quantum groups, Duke Math. J. 69 (1993), 455-485.

[19] M. Kashiwara, The crystal base and Littelmann's refined Demazure character formula, Duke Math. J. 71 (1993), 839-858.

[20] M. Kashiwara, Similarity of crystal bases, Contemp. Math. 194 (1996), 177-186.

[21] K. Kaveh, Crystal bases and Newton-Okounkov bodies, Duke Math. J. 164 (2015), 2461-2506.

[22] K. Kaveh and A. G. Khovanskii, Convex bodies and algebraic equations on affine varieties, preprint 2008, arXiv:0804.4095v1; a short version with title Algebraic equations and convex bodies appeared in Perspectives in Analysis, Geometry, and Topology, Progr. Math. Vol. 296, Birkhäuser, 2012, 263-282.

[23] K. Kaveh and A. G. Khovanskii, Newton-Okounkov bodies, semigroups of integral points, graded algebras and intersection theory, Ann. of Math. 176 (2012), 925-978.

[24] V. Kiritchenko, Newton-Okounkov polytopes of flag varieties, preprint 2015, arXiv:1506.00362v2, to appear in Transform. Groups.

[25] S. Kumar, Kac-Moody Groups, their Flag Varieties and Representation Theory, Progr. Math. Vol. 204, Birkhäuser, 2002.

[26] R. Lazarsfeld and M. Mustata, Convex bodies associated to linear series, Ann. Sci. de I'ENS 42 (2009), 783-835.

[27] G. Lusztig, Canonical bases arising from quantized enveloping algebras, J. Amer. Math. Soc. 3 (1990), 447-498.

[28] G. Lusztig, Quivers, perverse sheaves, and quantized enveloping algebras, J. Amer. Math. Soc. 4 (1991), 365-421.

[29] G. Lusztig, Introduction to Quantum Groups, Progr. Math. Vol. 110, Birkhäuser, 1993.

[30] G. Lusztig, Semicanonical bases arising from enveloping algebras, Adv. Math. 151 (2000), 129-139.

[31] P. Littelmann, Cones, crystals, and patterns, Transform. Groups 3 (1998), 145-179.

[32] T. Nakashima, Polyhedral realizations of crystal bases for integrable highest weight modules, J. Algebra 219 (1999), 571-597.

[33] T. Nakashima, Polytopes for crystallized Demazure modules and extremal vectors, Comm. Algebra, 30 (2002), 13491367.

[34] S. Naito and D. Sagaki, Crystal bases and diagram automorphisms, in Representation Theory of Algebraic Groups and Quantum Groups, Adv. Stud. Pure Math. Vol. 40, Math Soc. Japan, Tokyo, 2004, 321-341.

[35] S. Naito and D. Sagaki, Crystal base elements of an extremal weight module fixed by a diagram automorphism, Algebr. Represent. theory 8 (2005), 689-707.

[36] T. Nakashima and A. Zelevinsky, Polyhedral realizations of crystal bases for quantized Kac-Moody algebras, Adv. Math. 131 (1997), 253-278.

[37] A. Okounkov, Brunn-Minkowski inequality for multiplicities, Invent. Math. 125 (1996), 405-411.

[38] A. Okounkov, Why would multiplicities be log-concave?, in The Orbit Method in Geometry and Physics, Progr. Math. Vol. 213, Birkhäuser, 2003, 329-347.

[39] D. Sagaki, Crystal bases, path models, and a twining character formula for Demazure modules, Publ. Res. Inst. Math. Sci. 38 (2002), 245-264.

[40] A. Savage, Quiver varieties and Demazure modules, Math. Ann. 335 (2006), 31-46.

[41] T. A. Springer, Linear Algebraic Groups, 2nd ed., Progr. Math. Vol. 9, Birkhäuser, 1998.

Department of Mathematics, Tokyo Institute of Technology, 2-12-1 Oh-OKayama, Meguro-ku, Tokyo 152 8551, JAPAN

E-mail address: fujita.n.ac@m.titech.ac.jp 